\title{
Systematic prioritization of candidate genes in disease loci identifies TRAFD1 as a master regulator of IFN $\gamma$ signalling in celiac disease
}

Adriaan van der Graaf ${ }_{1}^{\#}$, Maria Zorro ${ }^{\#}$, Annique Claringbould 1 , Urmo Vosa1,2, Raul AguirreGamboa1, Chan $\mathrm{Li}_{1,3}$, Joram Mooiweer 1 , Isis Ricano-Ponce 1 , Zuzanna Borek4,5, Frits Koning6, Yvonne Kooy-Winkelaar6, Ludvig Sollid3, Shuo-Wang Qia03, BIOS consortium,

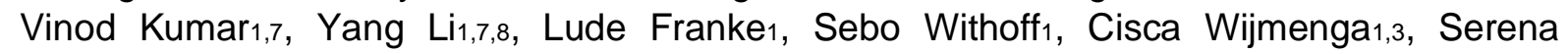
Sanna1, $9^{\#_{\star}}$, Iris Jonkers $1,3^{\#_{*}}$.

\section{Affiliations}

1 Department of Genetics, University Medical Center Groningen, University of Groningen, Groningen, the Netherlands

2 Estonian Genome Center, Institute of Genomics, University of Tartu, Tartu, Estonia 3 3 Department of Immunology, K.G. Jebsen Coeliac Disease Research Centre, University of 3 Oslo, Oslo, Norway

4 Deutsches Rheumaforschungszentrum Berlin (DRFZ), An Institute of the Leibniz Association, Berlin, Germany

5 Charité - Universitätsmedizin Berlin, corporate member of Freie Universität Berlin, 7 Humboldt-Universität zu Berlin, and Berlin Institute of Health, Department of 8 Gastroenterology, Infectious Diseases and Rheumatology, Berlin, Germany 6 Department of Immunohematology and Blood Transfusion, Leiden University Medical 20 Center, Leiden, the Netherlands

17 Department of Internal Medicine and Radboud Center for Infectious Diseases (RCI), 22 Radboud University Medical Center, Nijmegen, the Netherlands

23 Department of Computational Biology for Individualised Infection Medicine, Centre for 24 Individualised Infection Medicine, Helmholtz Centre for Infection Research, Hannover 25 Medical School, Hannover, Germany

9 Istituto di Ricerca Genetica e Biomedica (IRGB) del Consiglio Nazionale delle Ricerche 27 (CNR), Monserrato, Italy

\# These authors contributed equally

${ }^{*}$ Corresponding Authors 


\section{Abstract}

Background: Celiac disease $(\mathrm{CeD})$ is a complex $\mathrm{T}$ cell-mediated enteropathy induced by gluten. Although genome-wide association studies have identified numerous genomic regions associated with $\mathrm{CeD}$, it is difficult to accurately pinpoint which genes in these loci are most likely to cause CeD.

Results: We used four different in silico approaches - Mendelian Randomization inverse variance weighting, COLOC, LD overlap and DEPICT - to integrate information gathered from a large transcriptomics dataset. This identified 118 prioritized genes across $50 \mathrm{CeD}$ associated regions. Co-expression and pathway analysis of these genes indicated an association with adaptive and innate cytokine signalling and T cell activation pathways. 51 of these genes are targets of known drug compounds and likely druggable genes, suggesting that our methods can be used to pinpoint potential therapeutic targets. In addition, we detected 172 gene-combinations that were affected by our CeD-prioritized genes in trans. Notably, 41 of these trans-mediated genes appear to be under control of one master regulator, TRAFD1, and were found to be involved in IFN $\gamma$ signalling and $\mathrm{MHC} I$ antigen processing/presentation. Finally, we performed in vitro experiments that validated the role of TRAFD1 as an immune regulator acting in trans.

Conclusions: Our strategy has confirmed the role of adaptive immunity in CeD and revealed a genetic link between $\mathrm{CeD}$ and the IFN $\gamma$ signalling and $\mathrm{MHC}$ I antigen processing pathways, both major players of immune activation and CeD pathogenesis.

Key words: Celiac disease, gene prioritization, expression quantitative trait loci (eQTL) 
60

61

\section{Glossary}

Underlined words are definitions that have been explained in the preceding lines.

eQTL - expression quantitative trait locus, a location on the genome that is statistically associated to changes in gene expression.

cis-eQTL - an eQTL located in the same locus of the gene that is being interrogated (within $1.5 \mathrm{Mb}$ from gene transcript start or end).

trans-eQTL - an eQTL that is not physically close to the gene that is being interrogated (>1.5Mb from transcript start/end or on a different chromosome).

cis-eQTL gene - a gene that is associated with a change in expression as a consequence of a cis-eQTL.

trans-eQTL gene - a gene that is associated with a change in expression as a consequence of a trans-eQTL.

CeD - celiac disease

CeD-associated region - a genomic region that is associated to CeD based on results from genome-wide association studies on CeD.

Prioritized gene - a gene prioritized as being potentially causal for $\mathrm{CeD}$ according to the four statistical methods depicted in Figure 1A-B. In this study, prioritized genes are always within the CeD-associated regions.

Mediating cis gene - a prioritized gene that is statistically responsible for the change in expression of a trans-eQTL gene. Of note, while the trans-eQTL is located in the same CeDassociated region of the mediating cis-gene, the mediated trans-gene is not.

Mediated trans gene - a gene located outside CeD-associated regions that is statistically mediated by a mediating cis gene located in the same region of the corresponding transeQTL. 


\section{Introduction}

Celiac disease $(\mathrm{CeD})$ is an auto-immune disease in which patients experience severe intestinal inflammation upon ingestion of gluten peptides. $\mathrm{CeD}$ has a large genetic component, with heritability estimated to be approximately $75 \% 1$. The largest CeD-impacting locus is the HLA region, which contributes approximately $40 \%$ of CeD heritability2. While the individual impacts of CeD-associated genes outside the HLA region are smaller, they jointly account for an additional $20 \%$ of heritability. Previous genome-wide association studies (GWAS) have identified 42 non-HLA genomic loci associated with $\mathrm{CeD}_{3-6}$, but the biological mechanisms underlying the association at each locus and the genes involved in disease susceptibility are largely unknown. Yet, identification of these non-HLA genetic components and an understanding of the molecular perturbations associated with them are necessary to understand CeD pathophysiology.

Understanding the biological mechanisms of non-HLA CeD loci is difficult: only three of these loci point to single nucleotide polymorphisms (SNPs) located in protein-coding regions3. The other CeD-risk loci cannot be explained by missense mutations, making it necessary to look at other biological mechanisms such as gene expression to explain their role in $\mathrm{CeD}$ pathogenicity. Several studies have been performed to integrate expression quantitative trait loci (eQTLs) with CeD GWAS associations 4,7,8, and several candidate genes, including UBASH3A, CD274, SH2B3 and STAT49, have been pinpointed, implicating $\mathrm{T}$ cell receptor, $\mathrm{NF} \kappa \mathrm{B}$ and interferon signalling pathways as biological pathways associated with CeD pathology. Unfortunately, these eQTL studies had limited sample sizes, which reduced their power to identify cis- and (especially) trans-eQTLs. Furthermore, previous attempts to integrate eQTLs have mostly annotated genomic loci based on catalogued eQTLs without formally testing the causality of the genes in the onset or exacerbation of $\mathrm{CeD}_{8,10,11 \text {. }}$

Gene expression and GWAS data can also be integrated using methodologies that identify shared mechanisms between diseases. These methods can be roughly divided into three classes: variant colocalization methods, causal inference methods and co-expression methods. Colocalization methods consider the GWAS and eQTL summary statistics at a locus jointly and probabilistically test if the two signals are likely to be generated by the same causal variant12. Causal inference methods test if there is a causal relationship between expression changes and the disease, using genetic associations to remove any confounders 13,14. Finally, co-expression methods do not use eQTL information, but rather test if there is significant co-expression between the genes that surround the GWAS locus 15. 
119 Unfortunately, there is no current "gold standard" method for finding the causal gene behind 120 a GWAS hit, as all the methods discussed here are subject to their respective assumptions, 121 drawbacks and caveats. However, it is worthwhile to use all these methods in parallel to find 122 the most relevant causal genes for CeD.

Here, we systematically applied all four methods to the latest meta-analysis results for $\mathrm{CeD}_{5}$ and coupled them with eQTL results from the Biobank Integrative Omics Study (BIOS) cohort16, one of the largest cohorts for which there is genotype and RNA-seq expression data of peripheral blood mononuclear cells (schematic overview Fig. 1A-B). We focused on 58 GWAS loci that showed significant association with CeD at $p<5 \times 10_{-6}$. Our approach prioritized 118 genes in 50 loci and identified one gene, TRAFD1, as a master regulator of trans-effects. We then experimentally validated the role of TRAFD1-mediated genes using RNA-seq in a disease-relevant cell type. Our study yields novel insights into the genetics of $\mathrm{CeD}$ and is proof-of-concept for a systematic approach that can be applied to other complex diseases.

\section{Methods}

\section{Genotypes for eQTL analysis}

We used the BIOS cohort ${ }_{16}$ to map eQTLs in 3,746 individuals of European ancestry. The BIOS cohort is a collection of six cohorts: the Cohort on Diabetes and Atherosclerosis Maastricht17, the Leiden Longevity Study 18 , Lifelines DEEP ${ }_{19}$, the Netherlands Twin Registry20, the Prospective ALS Study Netherlands21 and the Rotterdam Study22. As described in Vosa et al.23, each cohort was genotyped separately using different arrays. Genotypes were subsequently imputed to the Haplotype Reference Consortium panel (HRC v1.0) on the Michigan imputation server 24.

We considered only biallelic SNPs with a minor allele frequency (MAF) $>0.01$, a Hardy-Weinberg test $p$ value $>10^{-6}$ and an imputation quality RSQR $>0.8$. To remove related individuals, a genetic relationship matrix (GRM) was created using plink $1.9_{25}$ (command -make-grm-bin) on linkage disequilibrium (LD)-pruned genotypes (option: "-indep 5052 "). Pairs of individuals with a GRM value $>0.1$ were considered related, and one individual was removed from each of these pairs. Population outliers were identified using a principal component analysis on the GRM, and we removed individuals who were more than 


\section{Expression quantification}

We used the same procedure for RNA gene expression control and processing as described in Zhernakova et al.16 In brief, RNA was extracted from whole blood and paired-end sequenced using the Illumina HiSeq 2000 machine. Read alignment of RNA-seq reads was done using STAR (v2.3.0)26 using a reference genome with masked variants with MAF < 0.01 in the Genome of the Netherlands27. Aligned reads were quantified using HTSeq28. Samples were removed if they had fewer than $80 \%$ aligned reads, fewer than $85 \%$ exonmapping reads, or if they had a median 3' bias larger than $70 \%$ or smaller than $45 \%$. Unobserved expression confounders were removed following the procedure of Zhernakova et al.16, correcting the expression matrix for the first 25 principal components as well as 3' bias, 5 'bias, GC content, intron base pair percentage and sex.

\section{eQTL analysis}

After genotype and RNA-seq quality controls (QCs), 3,503 individuals, 19,960 transcripts and 7,838,327 autosomal SNPs remained for analyses. We performed genome-wide eQTL mapping for the transcripts using plink $1.9_{25}$ with the --assoc command. We defined ciseQTL variants as those located within \pm 1 .5Mb of the transcript and trans-eQTLs as variants located outside these boundaries. To select variants that could explain the cis-eQTL signal of a gene, we used GCTA-COJO software29 v1.26. For this analysis, we required selected variants to reach a $p$-value threshold of $5 \times 10-6$ and included the BIOS cohort genotypes as LD reference. This identified 707 genes with at least one eQTL reaching this threshold, 357 of which had more than one conditionally independent eQTL variant.

\section{CeD summary statistics associated regions and candidate genes}

We used summary statistics from a CeD GWAS meta-analysis of 12,948 cases and 14,826 controls that analysed 127,855 variants identified using the Immunochip array5. SNP positions were lifted over to human genome build 37 using the UCSC liftover tool. We first identified lead associated variants in the CeD meta-analysis by performing $p$-value clumping: we used plink $1.9_{25}$ to select variants at a $p$-value threshold of $5 \times 10-6$ and pruned variants in LD with these selected variants using standard plink settings $\left(R_{2}>0.5\right.$, utilizing 1000 Genomes European sample LD patterns)25,30. We removed variants in an extended HLA region (chromosome $6,25 \mathrm{Mb}$ to $37 \mathrm{Mb}$ ) due to the complex long range $\mathrm{LD}$ structure in this region and because we aim to understand the function of the non-HLA genetic component of CeD. We looked for candidate genes around the clumped variants as follows. First, we 
defined regions around every clumped variant by padding the clumped SNP position by $1 \mathrm{Mb}$ on both sides. We then joined all overlapping CeD-associated regions together and looked for gene transcripts that partly or fully overlapped with the associated regions. This approach identified $58 \mathrm{CeD}$-associated regions and 1,235 candidate genes that are potentially causal for CeD. Of note, the CeD-association windows were set to be smaller than the eQTL window so that $\mathrm{eQTL}$ associations would fully overlap the associated CeD GWAS peak even when a gene is on the edge of the CeD-associated region.

\section{Gene prioritization using Mendelian Randomization-Inverse Variance Weighting (MR- IVW), COLOC, LD overlap and DEPICT}

We prioritized CeD-associated genes using three eQTL-based methods - MR-IVW 31 , COLOC $_{12}$ and LD overlap - and one co-regulation-based method, DEPICT 15 . For the MRIVW method, we used the independent variants identified by GCTA-COJO as instrumental variables 13,32 to test causal relationships between changes in gene expression and CeD. MR-IVW was only performed when there were three or more independent eQTLs available (164 genes). A gene was significant for the MR-IVW test if the causal estimates passed a Bonferroni threshold $p$-value of $3.1 \times 10-4$. Heterogeneity of causal estimates was accounted for and corrected for using Weighted Median MR analysis and Cochran's $Q$ test 3 . For the COLOC method, we used the 'coloc' $R$ package and considered a gene significant for the COLOC analysis if the posterior probability of shared variants $(\mathrm{H} 4)$ was larger than 0.9 . For the LD overlap method, a gene was considered significant if there was high LD $\left(r_{2}>0.8\right)$ between the top independent $\mathrm{eQTL}$ and the top $\mathrm{CeD}$ variant in the region. Finally, we applied DEPICT 15 to the clumped CeD GWAS variants described in 'CeD summary statistics associated regions and candidate genes'. Genes identified by the DEPICT analysis were considered significant if a False Discovery Rate $($ FDR $)<0.05$ was found with DEPICT's own permutation measure.

We scored each gene in the CeD-associated loci by considering each of the four prioritization methods. A gene was prioritized as 'potentially causal' in CeD pathology when one of the four methods was significant (one line of evidence). If multiple lines of evidence were significant, the gene was prioritized more highly than when only a single line of evidence was available.

To explore how the prioritized genes affect $\mathrm{CeD}$ risk, we gave each gene an effect direction based on the effect direction of the top variants in the eQTL and the CeD GWAS using the following algorithm: 
1. If there was a concordant effect that was significant in the top variants of both the eQTLs and the GWAS, the direction of the concordant effect was chosen.

2. If there was a concordant effect, but no significance of the SNP in one of the datasets, we could not be sure of an effect direction, and a question mark was chosen. The only exception to this was if the MR-IVW was significant, when we chose the direction of the MR-IVW effect.

3. If there was a discordant effect between the top SNPs, and both were significant in both datasets, a question mark was chosen. The only exception to this was when the IVW was significant, when the IVW effect was chosen.

4. If there was a discordant effect and there was significance in only one of the GWAS from the eQTL top SNP, the eQTL direction was chosen.

5. If there was a discordant effect and there was significance in only one of the eQTL from the GWAS top SNP, the GWAS direction was chosen.

6. If there was otherwise a discordant effect, a question mark was chosen.

Each gene is given a mark: positive ('+'), negative ('-') or unknown ('?'). '+' indicates that increased expression increases CeD risk. '-' indicates that increased expression decreases CeD risk. '?' indicates that it is unknown how the expression affects CeD risk.

\section{Co-regulation clustering}

The genes that have been prioritized may have some shared function in CeD pathology. To identify possible shared pathways, we performed co-regulation clustering analysis based on 1,588 normalized expression co-regulation principal components identified from RNA-seq information across multiple human tissues by Deelen et al 34 . We performed pairwise Pearson correlation of our prioritized genes with these 1,588 principal components and derived a correlation $Z$ score for each prioritized gene pair. We then performed hierarchical clustering of this $Z$ score matrix using Ward distances and identified 4 clusters from the resulting dendrogram.

\section{Trans $\mathrm{EQTL}$ and mediation analysis}

238 autosomal genes that were not located in, but were associated with, a significant transeQTL variant $(p<5 \times 10-8)$ in the CeD-associated regions were identified and used as potential targets for mediation by our associated genes in the CeD-associated loci (86 potential cis mediating genes). We first selected trans-eQTL genes that were co-expressed (Pearson $r>0.1,197$ gene combinations) with prioritized genes, then performed mediation analysis by running the trans-eQTL association again using the expression of the cis-eQTL gene as a covariate. We defined a trans-mediated gene if, after mediation analysis, the change (increase or decrease) in the effect size of the top trans-eQTL variant was significant 
according to the statistical test described in Freedman and Schatzkin 35 . For this analysis, we used a Bonferroni-adjusted $p$-value of $3.0 \times 10-4$.

\section{Cell type proportion and SH2B3 expression mediation analysis}

To assess if the cis-eQTL effect of TRAFD1 was not a proxy for cell-type composition, we performed mediation analyses in a fashion similar to the trans mediation analysis above using cell proportions measured in a subset of individuals in the BIOS cohort. To ensure that there was no residual effect of $S H 2 B 3$-expression on the mediating effect of TRAFD1, we corrected the original TRAFD1 expression levels for the expression levels of $S H 2 B 3$, leaving $T R A F D 1$ expression independent of $S H 2 B 3$, and reran the mediation analysis.

Literature review. We performed a REACTOME pathway 36 analysis to determine the potential function of the prioritized genes. This was complemented with a literature search (research and review papers) in Pubmed. For the coding and non-coding genes for which no studies were found, Genecards (www.genecards.org) and Gene Network v2.0 datasets (www.genenetwork.nl)34 were used, respectively. Information regarding the potential druggability of the prioritized genes was obtained from DrugBank 37 , the pharmacogenetics database 38 and a previous study that catalogued druggable genes 39.

THP-1 culture. The cell line THP-1 (Sigma Aldrich, ECACC 88081201) was cultured in RPMI 1640 with L-glutamine and 25mM HEPES (Gibco, catalogue 52400-025), and supplemented with $10 \%$ fetal bovine serum (Gibco, catalogue 10270) and 1\% penicillin/ streptomycin (Lonza, catalog DE17602E). The cells were passed twice per week at a density lower than $0.5 \times 10_{6}$ cells $/ \mathrm{ml}$ in a humidified incubator at $5 \% \mathrm{CO}_{2}, 37^{\circ} \mathrm{C}$.

siRNA treatment. THP- 1 cells were plated at $0.6 \times 10_{6}$ cells $/ \mathrm{ml}$ and transfected with $25 \mathrm{nM}$ siRNA using Lipofectamine RNAimax transfection reagent (Invitrogen, catalogue 13788), according to the manufacturer's protocol. Cells were treated with an siRNA to target TRAFD1 (Qiagen catalogue 1027416, sequence CCCAGCCGACCCATTAACAAT) (Knockdown (KD)), and cells treated with transfection mix without siRNA (Wild type (WT)) or non-targeting control siRNA (scrambled (SCR)) (Qiagen catalog SI03650318, sequence undisclosed by company) were included as controls. All the treatments were performed in triplicate. 72 hours after transfection, a small aliquot of cells was stained for Trypan Blue exclusion to determine cell viability and proliferation. The cells were stimulated with either LPS (10 ng/ml) from E. coli (Sigma catalogue 026:B6) or media alone (unstimulated) for 4h. Subsequently, the cells were centrifuged, and the cell pellets suspended in lysis buffer and stored at $-80^{\circ} \mathrm{C}$ until used for RNA and protein isolation. 
qPCR. The total RNA from THP-1 cells was extracted with the mirVana ${ }^{\mathrm{TM}}$ miRNA isolation kit (AMBION, catalogue AM1561) and subsequently converted to cDNA using the RevertAid H Minus First Strand cDNA Synthesis Kit (Thermo scientific, catalogue K1631). qPCR was done using the Syber green mix (Bio-Rad, catalogue 172-5124) and run in a QuantStudio 7 Flex Real-Time system (Applied Biosystems, catalogue 448598). Primer sequences to determine KD levels of TRAFD1 were 5' GCTGTTAAAGAAGCATGAGGAGAC and 3' TTGCCACATAGTTCCGTCCG. GAPDH was used as endogenous qPCR control with primers 5' ATGGGGAAGGTGAAGGTCG and 3' GGGGTCATTGATGGCAACAATA. Relative expression values of TRAFD1 were normalized to the endogenous control GAPDH and calculated using the $\triangle \triangle C T$ method, then given as a percentage relative to SCR expression levels.

Western blot (WB). Cell pellets from THP-1 cells were suspended on ice-cold lysis buffer (PBS containing 2\% SDS and complete protease inhibitor cocktail (Roche, catalog 11697498001)). Protein concentration of cell extracts was determined using the BCA protein kit (Pierce, catalog 23225). Proteins were separated on $10 \%$ SDS-polyacrylamide electrophoresis gel and transferred to a nitrocellulose membrane. After 1 hour of blocking with $5 \%$ fat-free milk in Tris-Tween-Buffer-Saline, the membranes were probed for 1 hour at room temperature with mouse mono-clonal TRAFD1 antibody 1:1000 (Invitrogen, catalog 8E6E7) or mouse monoclonal anti-actin antibody 1:5000 (MP Biomedicals, catalog 08691001), followed by incubation with goat anti-mouse horseradish peroxidase-conjugated secondary antibodies 1:10000 (Jackson Immuno Research, catalog 115-035-003). After three 10-minute washes, the bands were detected by Lumi light WB substrate (Roche, catalogue 12015200001) in a Chemidoc MP imaging system (Bio-Rad) and quantified using Image Lab $^{\mathrm{TM}}$ software (Bio-Rad). The band intensity of TRAFD1 was normalized to actin, and the TRAFD1 SCR control level was set as $100 \%$.

Statistical analysis for in vitro experiments in THP-1 cells. The statistical analyses of proliferation, qPCR and WB were performed using Prism 5 software (GraphPad Software, Inc.). Results are presented as mean \pm SEM from a representative experiment. Statistical differences were evaluated using a one-tailed $t$-test.

RNA sequencing (RNA-seq) in THP-1 cells. RNA from THP-1 cells was extracted with the mirVana ${ }^{\mathrm{TM}}$ miRNA isolation kit (AMBION, catalog AM1561). Prior to library preparation, extracted RNA was analysed on the Experion Stdsend RNA analysis kit (Bio-Rad, catalog 7007105). $1 \mu \mathrm{g}$ of total RNA was used as input for library preparation using the quant seq 3' kit (Lexogen, catalog 015.96), according to the manufacturer's protocol. Each RNA library was sequenced on the Nextseq500 (Illumina). Low quality reads, adaptors and poly-A tail 
reads were removed from FASTQ files. The QC-ed FASTQ files were then aligned to the human_g1k_v37 Ensembl Release 75 reference genome using HISAT default settings40, and sorted using SAMtools 41 . Gene-level quantification was performed by the featurecounts function of the RSubread R package v1.6.2 $2^{42}$. A modified Ensembl version $75 \mathrm{gtf}$ file mapping only to the last 5' 500 bps per gene was used as gene-annotation database to prevent counting of reads mapping to intra-genic A-repeats. Gene-level differential expression analysis between conditions was performed using the DESeq2 R package 43 after removing genes with zero counts. Differentially expressed genes (DEGs) were defined as genes presenting an absolute log2 fold change (|log2 FC|) $>1$ and an FDR $\leq 0.01$ across treatment (WT vs. SCR or KD unstimulated cells). To identify the genes responding to LPS stimulation, the DEGs between unstimulated samples and their respective stimulated sample were determined. Venn diagrams were used to depict the relationship between these genes. REACTOME pathway analyses were performed to identify biological processes and pathways enriched in different sets of DEGs using the enrichr API. Enrichments were considered significant if they were below a 0.05 FDR-threshold defined by the enrichr $\mathrm{API}_{36}$.

Gene set permutation analysis. It can be difficult to determine if a set of genes is 'on average' more or less differentially expressed due to co-expression between the genes within the set. To mitigate this, we performed a permutation test that considers the median absolute T statistic calculated by DESeq2 43 in the WT-SCR experiment as a null observation and compared this null observation with the SCR-KD experimental comparison. This allowed us to compare the expected differential expression of a set of genes, based on the WT-SCR comparison, with the observed differential expression of the same set of genes in the SCRKD comparison, while still incorporating the co-expression structure of the data. To do this, we randomly selected a same-sized set of genes 1,000,000 times in each relevant experiment (WT-SCR or SCR-KD), and determined the observed median absolute T statistic. We calculated a ratio of how often the permuted value is higher than the observed value. For example, the observations can be that $1 \%$ of permuted gene sets are more differentially expressed in the WT-SCR experiment, while only $0.01 \%$ of permuted genes sets are more differentially expressed in the SCR-KD experiment. Finally, we divide these values by one another, (percentage SCR-KD)/(percentage WT-SCR), to calculate a fold increase in differential expression. In the example given above, this indicates that the KD is 100 times $(0.01 / 1=100)$ more differentially expressed than expected.

Available RNA-seq datasets. Four available RNA-seq datasets were included to study the pattern of expression of prioritized genes. A brief description of each dataset is provided below. (GEO submission in process). 
Whole biopsy samples. Duodenal biopsies were obtained from 11 individuals $(n=6$ CeD patients and $n=5$ controls) who underwent upper gastrointestinal endoscopy (previously described)44. All individuals gave informed consent. To identify DEGs between patients and controls, a filter of $|\log 2 \mathrm{FC}|>1$ and FDR $\leq 0.05$ was applied using the DESeq2 R package.

Intra epithelial cytotoxic lymphocytes (IE-CTLS). CD8+ TCR $\alpha \beta$ IE-CTLs cell lines were isolated from intestinal biopsies and expanded for 12 days, as described previously45. Cells were left unstimulated (controls) or treated for 3 hours with IFN $\beta$ (300 ng/ml, Pbl Assay science, cat 11410-2), IL-15 (20 ng/ml, Biolegend, cat 570304) or IL-21 (3 ng/ml, Biolegend, cat 571204$)$ ( $n=8$ samples per condition, as previously reported $)_{44}$. Differential expression analysis between unstimulated cells and cytokine-treated IE-CTLs was performed using the $R$ package DESeq2. DEGs were defined as genes presenting a $|\log 2 \mathrm{FC}|>1$ and an FDR $\leq$ 0.05 between untreated controls and cytokine-treated samples.

Gluten specific (gs) CD4+ T cells. gsCD4+ T cell lines were generated from intestinal biopsies and expanded for 2 weeks, as reported previously46. Cells were stimulated for 3 hours with $2.5 \mu \mathrm{g} / \mathrm{ml}$ of anti-CD3 (Biolegend, catalog 317315) and anti-CD28 (Biolegend, catalog 302923) antibodies. Untreated cells were included as control. $\mathrm{N}=22$ samples per condition. DEGs were extracted with the DESeq2 package using the cut-off of $|\log 2 \mathrm{fc}|>1$ and FDR $\leq 0.05$ between unstimulated samples and controls.

Caco-2 cells. After 2 weeks of expansion in Transwells, the cells were treated with $60 \mathrm{ng} / \mathrm{ml}$ of IFN $\gamma$ (PeproTech) for 3 hours. Untreated cells were included as controls. RNA samples were extracted and further processed for RNA-seq (as described previously44). DEGs between control and stimulated cells were extracted with the DESeq2 $\mathrm{R}$ package using a cut-off of $|\log 2 \mathrm{FC}|>1$ and FDR $\leq 0.05$.

\section{Results}

\section{Gene prioritization identifies 118 likely causal CeD genes.}

To identify genes that most likely play a role in $\mathrm{CeD}$ (prioritized genes), we combined a recent genome-wide association meta analysis5 with (1) eQTLs derived from whole-blood transcriptomes of 3,503 Dutch individuals 16 and (2) a co-regulation matrix derived from expression data in multiple different tissues and 77,000 gene expression samples 15 . We selected 1,258 genes that were within $1 \mathrm{Mb}$ of the $58 \mathrm{CeD}$-associated non-HLA variant regions $(p<5 \times 10-6)$ (see Methods), and prioritized the genes that are the most likely causally related to $\mathrm{CeD}$ using four different gene prioritization methods: MR-IVW 13 , COLOC $_{12}$, LD overlap and DEPICT 15 (Fig. 1A-B) (Supplementary Table 1). 


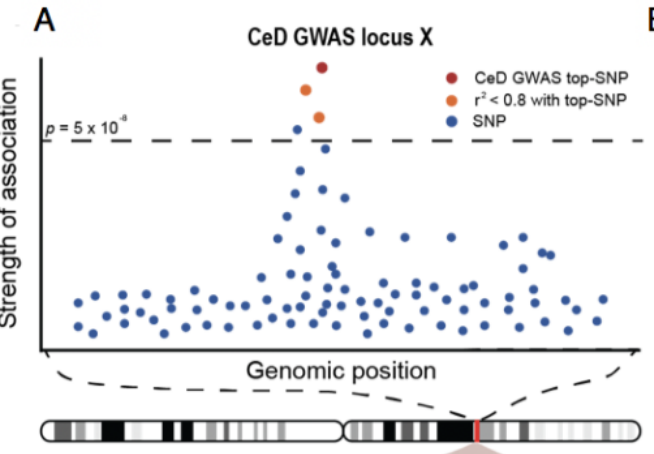

B
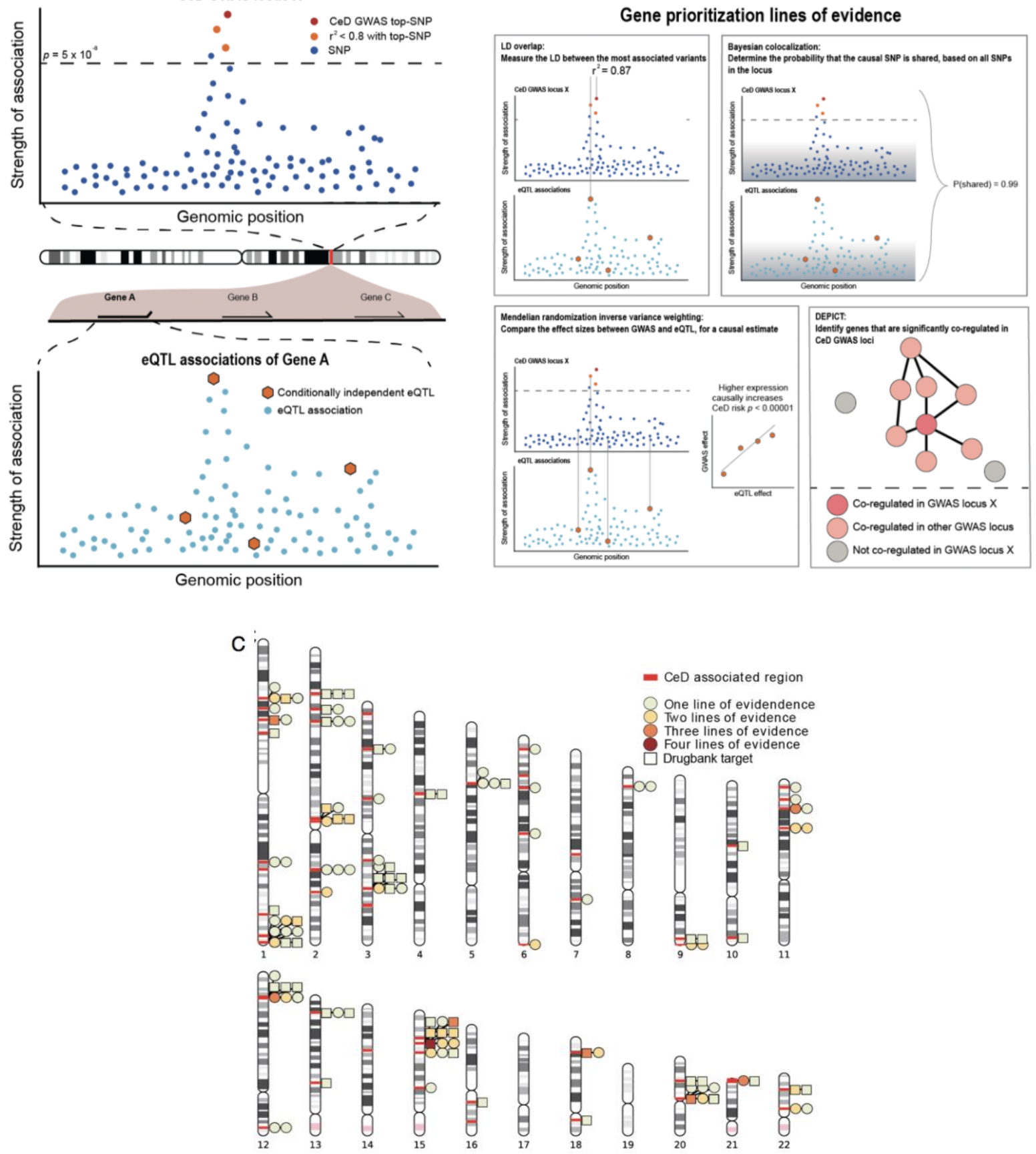

Fig. 1 Cis-eQTL prioritized candidate genes in CeD loci. (A) A CeD GWAS association curve at a hypothetical GWAS locus $X$ and the eQTL association at a potential candidate gene $A$. In both association plots, each dot represents a SNP plotted against the genomic position ( $\mathrm{X}$ axis) and the strength of association ( $Y$ axis). In the GWAS association curve, the top SNP is marked in red, while other SNPs above the significance threshold (dashed line) are coloured according to their LD with the top SNP. In the eQTL association curve, independent eQTLs are marked in red. (B) A conceptual depiction of the four statistical methods applied to link a disease locus to an eQTL locus. (C) A chromosome ideogram depicting the location of each prioritized gene identified in a CeD-associated GWAS locus. Loci are marked with red bars. Genes depicted by a square are the target of an approved drug or a drug in development. All other genes are depicted by a circle. Each circle or square is coloured according to the lines of evidences supporting its causal role. 
The first method we applied, MR-IVW, is a two-sample Mendelian Randomization approach called inverse variance weighting (see Methods). Our MR-IVW used summary statistics from two datasets: the eQTL and CeD GWAS. First, independent eQTLs at a locus were identified (see Methods), then the effect sizes of the eQTL and the GWAS were combined to identify gene expression changes that are causal (or protective) for $\mathrm{CeD}_{13,14}$ (see Methods). We only applied this method to a subset of 162 genes for which at least three independent cis-eQTL variants (at $p<5 \times 10-6$ ) were identified (see Methods) 32 . We accounted for heterogeneity using the $Q$ test and weighted median method and found that the effect sizes were very similar before and after correction (Supplementary Table 2).

The second method, COLOC, is a variant colocalization test in which we used eQTL and CeD summary statistics for all the SNPs in a locus and Bayesian probability to infer whether the $\mathrm{eQTL}$ and the CeD-association signals are likely to originate from the same causal variant 12 .

The third method, LD overlap, is a more classical annotation-type approach that prioritizes a gene if the top eQTL is in strong LD ( $\left.r_{2}>0.8\right)$ with the variant most significantly associated with $\mathrm{CeD}$ in a locus. This and the COLOC method were applied to 707 genes for which at least one significant $\mathrm{QQTL}$ variant was found.

Finally, we used DEPICT 15 , a gene-prioritization method based on co-regulation in expression datasets across multiple different tissues. DEPICT identifies enrichment for coregulated genes from genes in a GWAS locus. In contrast to the other methods, DEPICT assessed the potential role of all 1,258 genes independently of the presence of an eQTL.

In total, 118 out of the 1,258 assessed genes were prioritized by at least one of the four methods. Of these 118 genes, 28 had two lines of evidence, 7 genes (CD226, NCF2, TRAFD1, HM13, COLCA1, CTSH, UBASH3A) had three lines of evidence, and one gene (CSK) was supported by all four methods (Supplementary Table 1) (Fig. 1C). Overall, we identified potentially causal genes in 50 out of $58 \mathrm{CeD}$-associated regions.

The four different gene prioritization methods complement each other in different ways. DEPICT prioritized the most genes: 66 in total, 38 of them uniquely prioritized (38/66, $58 \%$ unique). One reason for this is that DEPICT is based on co-expression, not genetic background. Indeed, 16 genes prioritized by DEPICT do not have a significant eQTL associated with them. Overall, the most concordance was found between COLOC and LD overlap ( $30 \%$ and $26 \%$ unique genes, respectively) as these methods are the most similar, while MR-IVW uniquely prioritized a relatively large proportion of genes (9/20, 45\% unique). Thus, each method helps prioritize genes with multiple lines of evidence, but also adds a unique set of genes based on the assumptions of the method.

To see if any of these genes could lead to therapeutic intervention in CeD, we searched for the CeD-associated genes in DrugBank and assessed their druggability 
potential following Finan et al.39 (Supplementary Table 3). 26 of the 118 prioritized genes encode proteins that are targeted by an approved drug or a drug in development according to drugbank (Fig. 1C) (Supplementary Table 3). For example, drugs such as Natalizumab and Basiliximab that target the proteins encoded by ITGA4 and IL21R, respectively, are currently approved or under study for the treatment of immune-mediated diseases including rheumatoid arthritis47, Crohn's disease 48 and multiple sclerosis 49 or as an immunesuppressor to avoid kidney transplant rejection. An additional 25 genes encode proteins that are similar to proteins targeted by already approved drugs following Finan et al.39 (Supplementary Table 3).

\section{Co-expression patterns of cis-eQTL-prioritized loci reveal four functional clusters}

The biological function for the 118 prioritized genes and their role in CeD pathology is not fully understood. We sought to infer biological function using a guilt-by-association coregulation approach to identify clusters of shared molecular function (see Methods). We identified co-regulated genes by correlating our prioritized gene list in 1,588 principal components that were identified from the co-expression of 31,499 RNA-seq samples across multiple tissues 34 (Fig. 2A). We then performed REACTOME 2016 gene set enrichment 36 analysis to investigate the biological processes enriched in each cluster (Supplementary Table 4) (Supplementary Table 5).

We could not identify a specific biological process linked to our first co-regulation cluster. However, genes such as ULK3 (relevant for autophagy50) and CSK (relevant to T cell receptor (TCR) signaling 51 ) are included in this co-regulation cluster. Our second cluster encompasses genes (e.g. STAT1, CD274 and IL12A) implicated in interferon gamma (IFN $\gamma$ ) signalling and interleukin (IL)-6 signalling. Co-regulation cluster 3 contains genes (e.g. CD28, CTLA4 and ICOS) associated with co-stimulation by CD28, a process that is essential for modulating $T$ cell-activation. Finally, co-regulation cluster 4 contains chemokine (e.g. CCR1, CCR2 and CCR3) and cytokine signalling genes (e.g. IL2RA, IL21 and IL18R1) (Fig. 2B). The biological processes overrepresented in these co-regulation clusters are essential for the activation and function of the adaptive and innate immune system, which confirms and extends previous findings that implicate both arms of the immune system in $\mathrm{CeD}$ disease pathogenesis. Approximately $10 \%$ of the prioritized genes are long non-coding RNAs (IncRNAs) rather than protein-coding genes (Supplementary Table 1). Although little is known about the function of IncRNAs, their co-regulation pattern with the genes in clusters 2 and 4 suggests that they may be associated with cytokine/chemokine signalling (Fig. 2A, B). Moreover, by using Genenetwork34, we found that the IncRNAs RP3-395M20.9, AC007278.2 and AC104820.2 may be involved in tumour necrosis factor (TNF) signalling, neutrophil degranulation and chemokine receptor signalling, respectively, implying a role for these uncharacterized IncRNAs in immune regulation in CeD. 
A
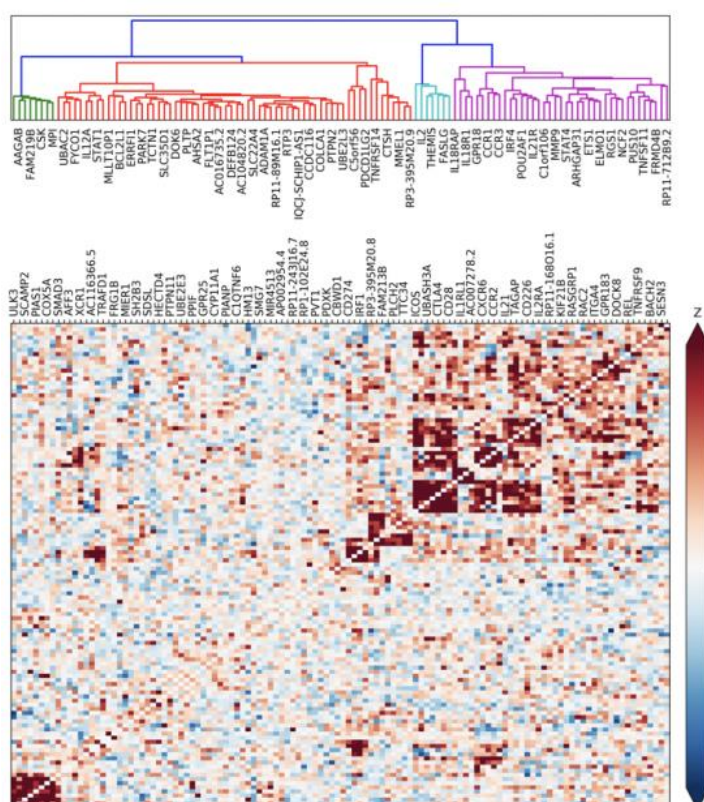
performed using the "average" method in hclust().
B
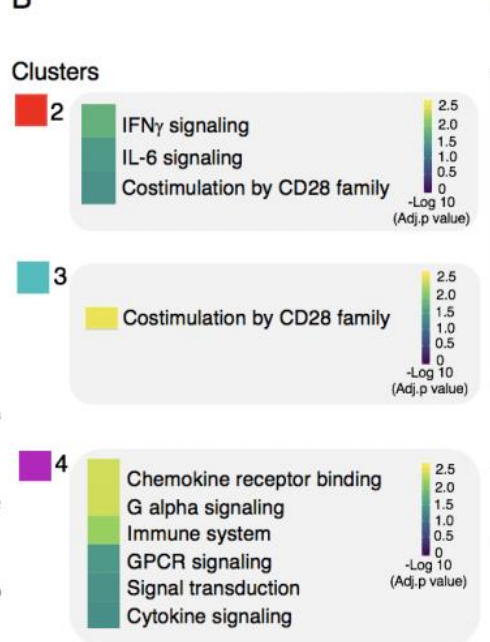

C

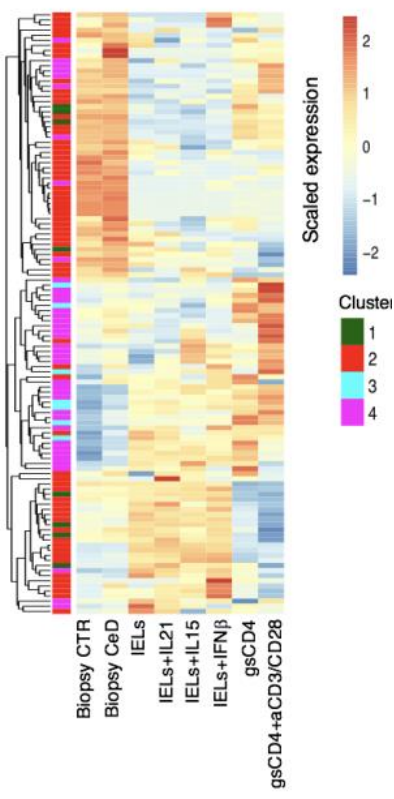

Fig. 2 Co-expression pattern of cis-eQTL prioritized genes reveals four functional clusters. (A) Heatmap showing the Spearman correlations between gene expression patterns of each prioritized gene. Blue squares indicate negative correlation. Red squares indicate positive correlation. Both are shaded on a gradient scale according to the $Z$ score of the correlation. A dendrogram computed with Ward distances between the correlations is shown on top of the heatmap. Branches of the dendrogram are coloured differently to mark separate clusters. (B) Results of the REACTOME gene set enrichment analysis of the genes belonging to each of the clusters identified in (A). Colour key denotes the significance (-log 10 multiple testing adjusted $p$ value) of each biological pathway. (C) Heatmaps depicting the scaled expression of prioritized genes belonging to the four clusters identified in (A) in three available RNA-seq datasets: intestinal biopsies from controls (CTR, $n=5$ samples) or CeD patients (CeD, $n=6$ samples); CD8+ TCR $\alpha \beta$ intraepithelial cytotoxic lymphocytes (IE-CTLs) unstimulated or treated with IL-21, IL-15 or IFN $\beta$ for 3 hours ( $n=8$ samples per condition) and gsCD4+ T cells unstimulated or treated with anti-CD3 and anti-CD28 (aCD3) for 3 hours ( $n=22$ samples per condition). Clustering was

\section{CeD candidate genes operate in immune and intestinal epithelial cells}

To complement our REACTOME gene set enrichment analysis and dig deeper into the biological processes and cell types in which the prioritized genes may act, we analysed their expression profiles in available RNA-seq datasets from disease-relevant cell types including 1) small intestinal biopsies of active CeD patients and healthy controls, 2) intra-epithelial cytotoxic lymphocytes (IE-CTLs) stimulated with disease-relevant cytokines IL-21, IL-15 and IFN $\beta$, and 3 ) gluten specific CD4+ T cells (gsCD4+ T cells) stimulated with antiCD3-antiCD28, which mimics the disease-specific response to gluten peptides (Fig. 2C) (differentially expressed genes for each dataset are available in Supplementary Table 6). We observed that the genes grouped in co-regulation clusters 1 and 2 are highly expressed in small intestinal biopsies and IE-CTLs, which is in line with the IFN $\gamma$ pathway enrichment seen in co-regulation cluster 2 (Fig. 2B). IFN $\gamma$ is mainly produced by gsCD4+ T cells and IE-CTLs and is known to disrupt the integrity of the intestinal epithelial cells in CeD-associated villous 
atrophy52-54. Within this cluster we also found genes specific to antigen-presenting cells (B cells, monocytes and dendritic cells) and epithelial cells such as IL12A and COLCA1, which are most expressed in small intestinal biopsies (Fig. 2C). The genes in co-regulation clusters 3 and 4 are highly expressed in gsCD4+T cells, especially after stimulation with antiCD3-antiCD28, indicating that these prioritized genes may be biologically relevant in the immediate $T$ cell receptor response to gluten ingestion.

The gene expression pattern of the prioritized genes, when combined with information from our literature search, suggests that these genes may control general biological processes (e.g. apoptosis, gene regulation and cytoskeleton remodelling) as well as specific immune functions (e.g. cell adhesion, cell differentiation and TCR signalling) in diverse cell types (e.g. T cells, neutrophils, B cells, monocytes, epithelial cells) (Fig. 3 and

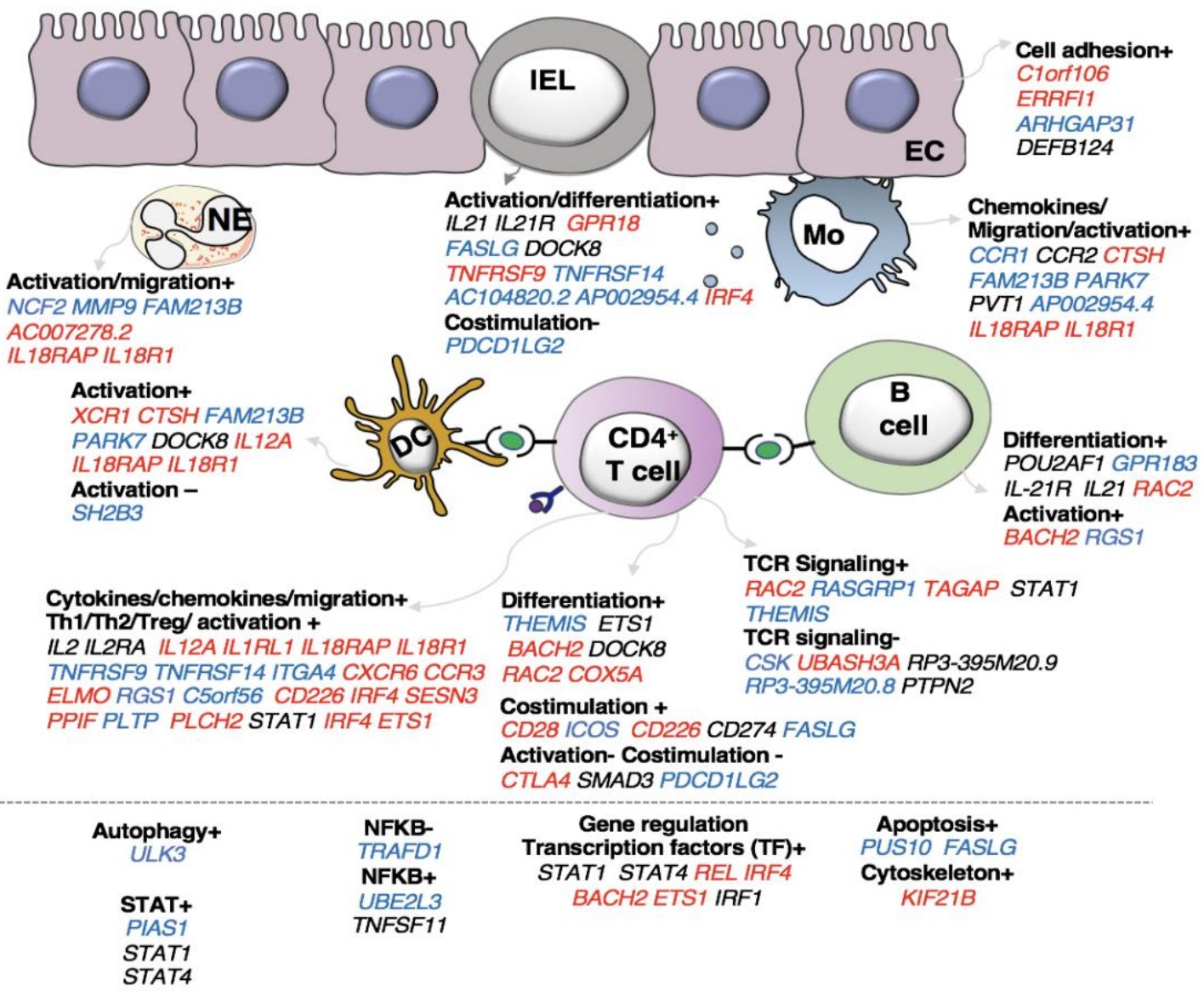

Fig. $3 \mathrm{CeD}$ candidate genes operate in immune cells and intestinal epithelial cells. Functions and cell types highlighted by the prioritized genes, according to our literature review (see Methods) ( $n=118$ genes, for 37 genes neither a function nor a specific cell type on which the gene may operate could be specified). All genes contributing to a specific function are listed under the sub-heading and coloured according to the change that leads to increased CeD risk: increased expression (red), decreased expression (blue), or undefined (black). The symbols + or - denote if a biological process is thought to be induced or repressed by the gene, respectively, according to literature. 


\section{Mediation analysis uncovers TRAFD1 as a major trans-eQTL regulator}

To further understand the potential regulatory function of the prioritized genes, we identified downstream regulatory effects by performing a trans-mediation analysis using a two-step approach (Methods) (Supplementary Fig. 1A). We first considered all genes with a transeQTL $(p<5 \times 10-8$ ) located in any of the 58 CeD-associated regions, then performed a mediation analysis by re-assessing the trans-eQTL effect after adjusting the expression levels for the expression of the prioritized gene(s) in the same locus (Fig. 4A).

Of the 497 possible prioritized gene-trans-eQTL gene combinations, we found 172 that exhibited significant mediation effects. These combinations map to 13 associated regions and represent 21 unique mediating cis-eQTL genes and 79 unique mediated transeQTL genes (Supplementary Table 8). Among all the associated regions, the CeDassociated region on chromosome 12 contained the largest number of both cis-mediating genes $(\mathrm{N}=5)$ and trans-mediated genes $(\mathrm{N}=60)$. In this region, TRAFD1 mediated more trans genes than all of the other regional cis-regulators and also had the highest mediation impact (average Z-score difference in effect size between mediated and unmediated analysis = 2.79) (Methods) (Supplementary Table 8) (Supplementary Fig. 1B). Of note, the top eQTL variant of TRAFD1 is a missense variant in the nearby gene SH2B3. This missense variant has been associated to a number of complex traits, including blood cell types and platelets, and autoimmune diseases55,56. However, we found that cell-type composition did not affect the eQTL-association of TRAFD1 in our cohort ( $p>0.044$ for 24 different cell-type traits) (Methods) (Supplementary Table 9). To ensure that the mediated trans genes of TRAFD1 were not mediated by SH2B3, we corrected TRAFD1 expression levels for SH2B3 and re-ran the mediation analysis. Here we found that the mediating effect of TRAFD1 was still significant for all 41 genes found initially and that the median Z-score difference between mediated and unmediated was higher than that of $S H 2 B 3$, although it was slightly attenuated compared to the original TRAFD1 signal (Supplementary Table 10) (Supplementary Figure 1B). Based on these results, we conclude that TRAFD1 is a master regulator of gene expression changes in the associated region (Fig. 4B) (Supplementary Table 10).

Strikingly, three of the TRAFD1 trans-mediated genes - STAT1, CD274 and $P D C D 1 L G 2$ - are also prioritized cis-genes in their respective loci (Fig. 4B). These results suggest that the trans-mediated TRAFD1-effects may have an additional additive effect in these CeD-associated loci. 
A
B

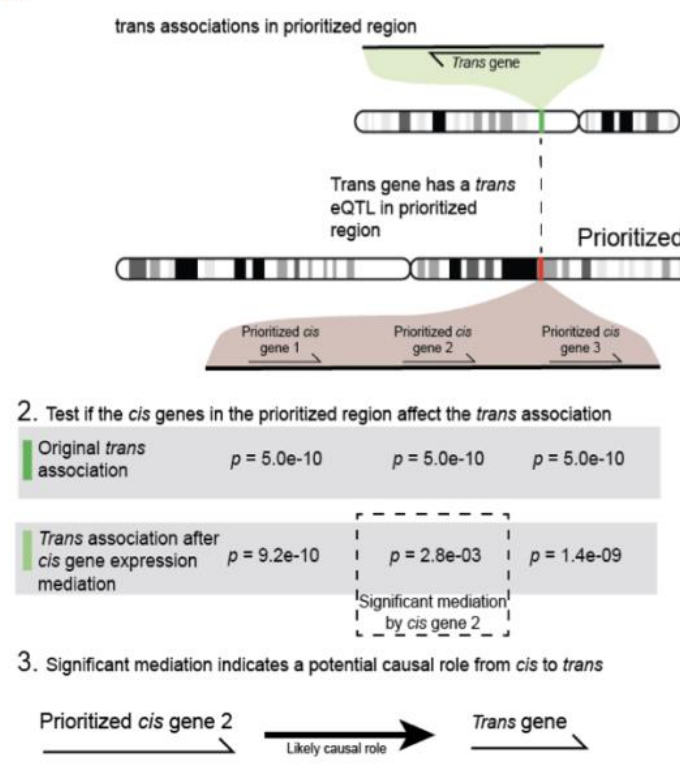

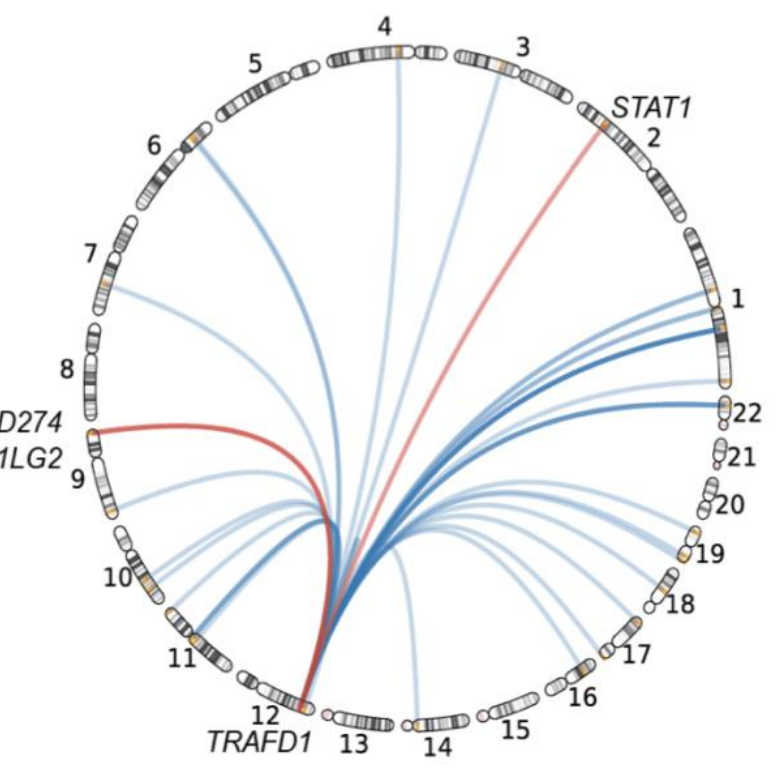

Fig. 4 Mediation analysis uncovers TRAFD1 as a major trans-eQTL regulator. (A) Workflow illustrating the main steps to identify trans-eQTL genes mediated by our cis-prioritized genes. First, we identified trans-eQTLs and trans genes that have a significant association $(p<5 \times 10-8)$ in our prioritized regions. Then, for every cis prioritized gene in the CeD-associated region, a mediation analysis was performed to determine if the cis gene expression explains the transeQTL effect. (B) Circular ideogram depicting the mediating effect of TRAFD1 on 41 trans genes. Three of the 41 trans-mediated genes were also prioritized by our cis-eQTL analysis (red).

TRAFD1 is a poorly characterized gene that has been suggested to act as a negative regulator of the NFKB pathway57. To further elucidate the biological processes in which the 41 TRAFD1 trans-mediated genes could be involved, we performed a REACTOME 2016 gene set enrichment analysis (Supplementary Table 11). Here we found that IFN $\gamma$ signalling, cytokine signalling and major histocompatibility complex class I $(\mathrm{MHCl})$ antigen processing / presentation are strongly enriched pathways, which points to a role for TRAFD1 and TRAFD1 trans-mediated genes in antigen presentation and immune response (Fig. 5A).

By looking into RNA-seq datasets from disease-relevant cell types, we noted that most TRAFD1 trans-mediated genes are upregulated in biopsies from patients with active CeD, and these genes include STAT1, CXCL10 and TAP1, which are essential for IFN response 58, chemotaxis59 and antigen processing60, respectively (Fig. 5B). Moreover, most TRAFD1 trans-mediated genes exhibit an increase in expression in response to IFN $\gamma$ in intestinal epithelial cells (Caco-2) or IFN $\beta$ in IE-CTLs (Fig. 5B). In contrast, antiCD3antiCD28 stimulation in gsCD4+T cells resulted in both up- and downregulation of the TRAFD1 trans-mediated genes, implying that TRAFD1 trans-mediated genes respond more strongly to IFN signalling (IFN $\gamma$ or IFN $\beta$ ) than to TCR activation by anti-CD3/anti-CD28. 
Indeed, the enrichment of the 41 TRAFD1 trans-mediated genes in significantly differentially expressed genes in biopsies, IE-CTLs, epithelial cells and gsCD4+Tcells was strongest in IECTLs and epithelial cells upon IFN signalling (Supplementary Table 6). Overall, our results suggest that TRAFD1 and TRAFD1 trans-mediated genes modulate IFN signalling upon antigen presentation, possibly via regulation of $\mathrm{NF} \kappa \mathrm{B}$, in $\mathrm{CeD}$ pathology.

A

IFN $\gamma$ signaling IFN signaling Cytokine signaling Immune system $\mathrm{MHCl}$ antigen processing \& presentation Adaptive immune system
B

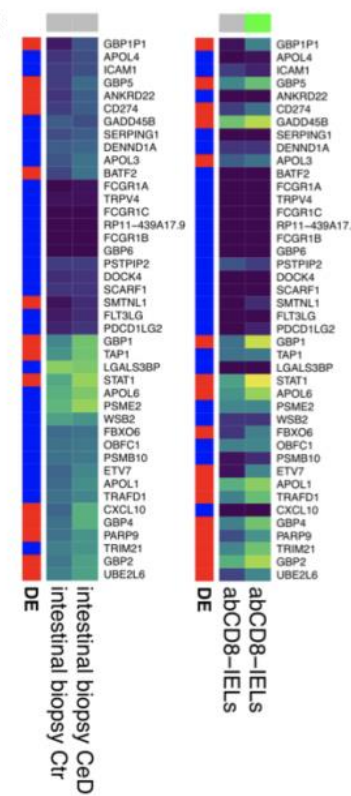

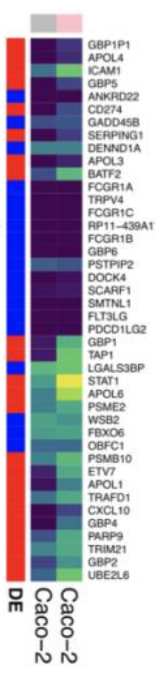

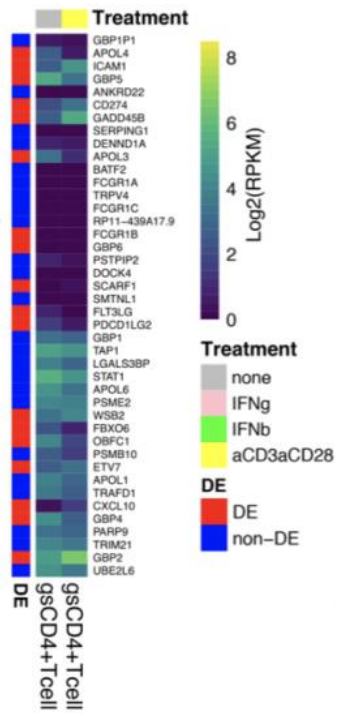

Fig. 5 TRAFD1 is a regulator of IFN $\gamma$ signalling genes. (A) Results of the REACTOME gene set enrichment analysis of TRAFD1-mediated genes ( $\mathrm{n}=41$ genes). Colour code denotes the significance (-log 10 adjusted $p$ value) of each biological pathway. (B) Unscaled heatmaps depicting the expression of these genes in RNA-seq datasets from different cell types: whole biopsies from controls (Ctr, $n=5$ samples) of $\mathrm{CeD}$ patients (CeD, $n=6$ samples); intraepithelial cytotoxic lymphocytes (IE-CTLs) unstimulated or treated with IFN $\beta$ for 3 hours $(n=8$ samples per condition); and Caco-2 cells untreated or stimulated with IFN $\gamma$ for 3 hours ( $n=8$ samples per condition). Red indicates that a gene is differentially expressed (DE), blue indicates that a gene is not differentially expressed (non-DE) (FDR $<0.01$ and $|\log 2(\mathrm{RPKM})>1|)$. Grey (none or unstimulated), pink (IFN $\gamma$ ), green (IFN $\beta$ ) and yellow (antiCD3/antiCD28) colours indicate the type of stimulation (treatment).

\section{TRAFD1 KD affects immune-activation genes}

We performed a siRNA KD experiment on TRAFD1 to gain more insights into the biological function of this gene and to independently validate the TRAFD1 trans-mediated genes. We also evaluated the transcriptional changes of knocking down TRAFD1 in the monocyte-like cell line THP-1 under resting conditions (unstimulated) or in the presence of LPS, a known inducer of the NFkB pathway61. 
After siRNA treatment, we observed no significant differences in cell viability or proliferation among the controls (WT and SCR) and the KD treatment (Supplementary Fig. 2A, B). However, as expected for the KD cell line, we noted a significant reduction in the expression of TRAFD1 compared to the controls in WB and qPCR analyses (Supplementary Fig. 2C-E). KD of TRAFD1 was also confirmed in the RNAseq data, with TRAFD1 expression levels reduced by $41 \%$ in unstimulated KD cells compared to unstimulated SCR cells (adjusted $p=0.004$ ) and by $34 \%$ in LPS-stimulated KD cells compared to LPS-stimulated SCR cells (not significant) (Supplementary Table 12). The reduced KD effect upon LPS stimulation is consistent with our expectation that TRAFD1 acts as negative regulator of the NFKB pathway, which is activated by several stimuli, including LPS 61 . Thus, the KD was successful and neither the transfection method nor a reduced expression of TRAFD1 had a toxic effect (Supplementary Fig. 2A-E).

Next, we tested if the 41 TRAFD1 trans-mediated genes were more differentially expressed than expected after LPS stimulation (Supplementary Fig. 3). To disentangle differential expression from the co-expression inherently present in a gene expression dataset, we devised a permutation scheme that compared the control (WT vs. SCR) observations with the KD (SCR vs. KD) observations (see Methods). This scheme takes into account the co-expression of a gene set, as this co-expression is present in both the control and the experimental observation. After performing 1,000,000 permutations of 42 genes (41 trans mediated genes and TRAFD1) in the LPS-stimulated comparison, the median test statistic in the control observations was observed 54 times more often than in the KD observations ( $0.270 \%$ for WT-SCR vs. $0.005 \%$ for SCR-KD, Supplementary Fig. 4 ). This indicates that the 41 trans-mediated genes and TRAFD1 are 54 times more differentially expressed than expected. We did not find increased differential expression of the same gene set in the unstimulated condition $(1.120 \%$ for WT-SCR vs. $0.307 \%$ for SCRKD, Supplementary Fig. 4), indicating that TRAFD1 mainly regulates genes in an LPSstimulated state.

To identify the role of TRAFD1 in immune cells and processes, we compared gene expression changes in the unstimulated condition versus the LPS stimulated condition for each treatment (WT, SCR or KD) separately (Supplementary Fig. 2F). Differential expression analysis showed that 353 genes were uniquely upregulated and 330 genes uniquely downregulated after TRAFD1 KD treatment (Supplementary Fig. 2G, H). We found no REACTOME gene set enrichment for these unique KD genes. We found 500 upregulated and 433 downregulated genes that were differentially expressed in all three 

performed hierarchical clustering, this separated the two gene sets into two clusters: cluster 1 shows a decreased response of genes in the TRAFD1 KD group (LPS cluster 1, Fig. 6B, Supplementary Table 13) and cluster 2 displays an increased expression in the TRAFD1 KD cells under unstimulated conditions that persists after LPS stimulation (LPS cluster 2, that the genes in LPS cluster 1 are involved in immune-related processes (e.g. cytokine signalling, RIG/IMDA5 induction of IFN signalling and IFN signalling, Fig. 6D), whereas the genes in LPS cluster 2 are associated with the heat shock response, which has been shown to be activated as a consequence of immune activation or immune response to stress62 (Fig. 6E, Supplementary Table 13). Together, these results suggest that TRAFD1 is a regulator of immune activation and inflammation.

A

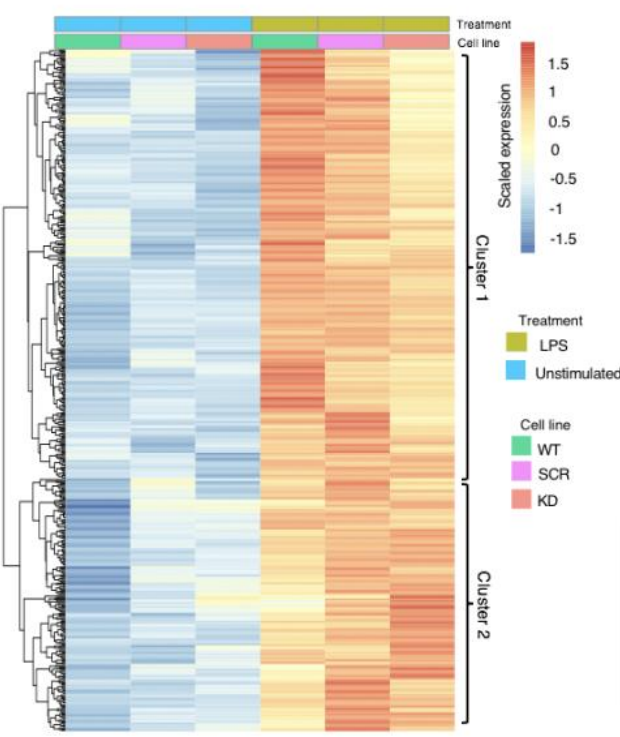

B

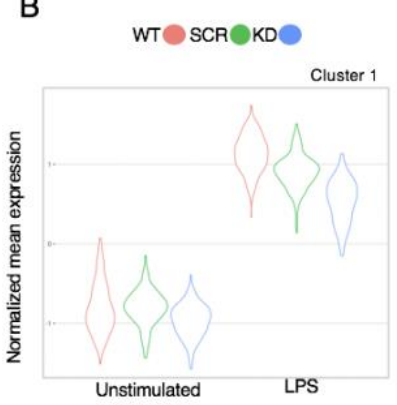

D

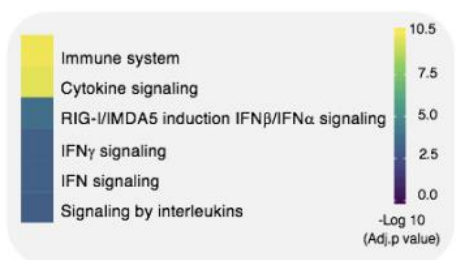

C

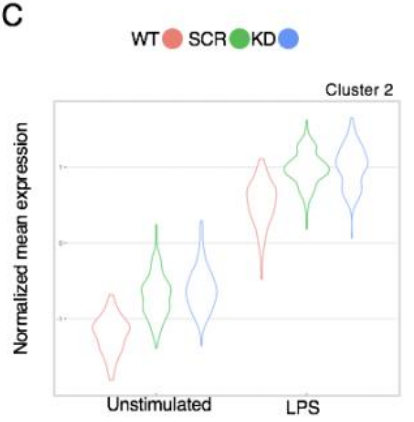

E

HSF1 dependent transactivation

Attenuation phase

Gene expression

Cellular response to heat

Cellular response to stress

HSF1 heat shock response
671
Fig 6. TRAFD1 knockdown affects immune activation and stress-related genes. (A) Heatmap showing the expression profile of the 500 shared DEGs identified in the knockdown experiments (see Methods and Supplementary Fig. 2 F, G). A dendrogram on the left of the heatmap depicts the strength of similarities based on Ward distance. (B, C) Violin plots showing the normalized gene expression of the genes belonging to the first and second cluster of DEGs identified in (A) in THP-1 cells under different experimental conditions (WT=untransfected, SCR=non-targeting siRNA, KD=siRNA targeting TRAFD1) and stimulations (LPS=lipopolysaccharide). (D, E) Results of REACTOME gene set enrichment analysis of the genes within the first (D) and second cluster (E). Significance (-log 10 adjusted $p$ value) of each biological pathway is indicated by the colour key. 


\section{Discussion}

685

686

687

688

689

690

691

692

693

694

695

696

697

698

699

700

701

702

703

704

705

706

707

708

709

710

711

712

713

714

715

716

717

718

In the present study we aimed to identify CeD candidate genes using four in silico methods (MR-IVW, COLOC, LD overlap and DEPICT) and whole blood transcriptomics data from a population-based cohort. While previous studies have used at least one of these methods $3,5,6,11$, to our knowledge this is the first effort that integrates the four different statistical approaches. This systematic prioritization approach resulted in 118 prioritized causal genes, including 26 that are direct targets of an approved drug or of drugs under development for other complex diseases, including autoimmune diseases. The coexpression pattern within a large RNA-seq dataset from blood 34 suggests these genes are involved in cytokine signalling in innate and adaptive cells as well as in T cell activation pathways. We also identified TRAFD1 to be trans-regulator of 41 genes, with a strong enrichment in IFN $\gamma$ signalling and MHC I antigen processing/presentation pathways, which are pivotal for the disease pathogenesis.

After clustering our cis-eQTL prioritized genes on shared co-regulation, we identified a cluster of genes involved in T cell activation and co-stimulation (cluster 3 ), highlighting a key role for $\mathrm{T}$ cell activation in the pathogenesis of $\mathrm{CeD}_{63}$. Within this co-regulation cluster we found the THEMIS, IL2, CD28, CTLA4 and UBASH3A genes (Fig. 2), whose functions include $\mathrm{T}$ cell differentiation and activation and the TCR macromolecular complex.

Another co-regulation cluster (cluster 4, Fig.2) grouped prioritized genes involved in cytokine and chemokine signalling events that affect the microenvironment during inflammation in the intestinal mucosa. For example, this group included CCR1 and CCR2, which control the activation and recruitment of inflammatory cells such as monocytes, dendritic cells and neutrophils64. IL21 was also included in this co-regulation cluster. This gene encodes IL-21, providing proliferation and survival signals to B cells65, which in turn produce the autoantibodies detected in $\mathrm{CeD}$ and could act as antigen-presenting cells for gsT cells, thus enhancing the inflammatory response66. Next to chemokine receptors, this co-regulation cluster contains cytokine receptor genes, e.g. IL18RAP and IL18R1. Which encode the IL-18 receptor, that is expressed in intestinal epithelial cells and mediates IL-18 controlled intestinal barrier integrity and immunity 67,68 . This cluster also contains transcription factors genes, e.g. IRF4, ETS1 and REL. IRF4 and ETS1 are essential for T helper 1 (Th1) differentiation69,70. Interestingly, gsCD4+ T cells exhibit a Th1 profile, that predominantly produce IFN $\gamma$, a cytokine that affects the integrity of the intestinal epithelial cells contributing to villous atrophy52-54. REL, that is also contained in cluster 4 , is a key regulator of NFKB signalling pathway, a major mediator of inflammation 71 , which is in line with the novel genetic association reported between NFאB and CeD by Ricano-Ponce et al.5 Moreover, CeD 
patients show a persistent activation of the $N F \kappa B$ pathway in the intestinal mucosa72 as well as a significant increase in the methylation level of 8 genes that belong to this pathway 73 . Thus, these results indicate that $\mathrm{CeD}$ patients present with a major defect in the NFKB signalling complex.

For practical reasons, most prioritization studies have been focused on incorporating cis-eQTLs74 and have mostly ignored trans-eQTLs, thus potentially missing long-distance co-regulated interactions74. In our study, we took advantage of a large transcriptomics cohort to run a trans mediation analysis for $\mathrm{CeD}$ loci. One of the most remarkable findings of this approach was that 41 trans-mediated genes were found to be controlled by a single gene: TRAFD1. These 41 genes are enriched for IFN $\gamma$ and MHC I antigen processing/presentation signalling pathways. Interestingly, gsCD4+ $T$ cells exhibit a Th1 profile and produce a large amount of IFN $\gamma$, one of the most predominant cytokines in $\mathrm{CeD}_{54}$. Some of the most striking effects of IFN $\gamma$ include induction of apoptosis in intestinal epithelial cells, alteration of intestinal permeability and activation of monocytes and dendritic cells, which may act as antigen-presenting cells for gsCD4+ T cells 75 .

TRAFD1 is thought to be a regulator of the NFאB signalling pathway 57 , suggesting that CeD-risk SNPs may modulate the NFKB complex via both cis and trans regulatory mechanisms. Our results also point to a role for TRAFD1 in response to IFN $\gamma$; however, IFN $\gamma$ does not typically activate NFKB signaling76 and the IFNG locus is not associated with CeD7. Thus, TRAFD1 may activate the production of other cytokines, which in turn activate the NFKB complex.

IE-CTLs, which are the effector cells in CeD, have not thus far been genetically associated with the disease. However, given that $\mathrm{MHC}-\mathrm{I}$ antigen presentation presentation/processing are essential for IEL activation and the striking activation of the 41 trans mediated genes in IE-CTLs upon IFN stimulation, we propose that the IE-CTLs are also genetically linked to the disease through the action of TRAFD1.

Despite the approaches implemented in our study to uncover the novel gene interactions and biological pathways that may underlie the disease, a major drawback is the limited genome coverage of the CeD summary statistics used in this study. These were derived from a GWAS that used the Immunochip platform, a genotyping platform that only measures genotypes in regions known to be associated with immune function. We thus acknowledge that our current interpretation of CeD loci is biased toward immune-related mechanisms. Only when comprehensive whole-genome $\mathrm{CeD}$ association analyses have been performed will we have an unbiased understanding of the disease pathophysiology. 
In our gene prioritization we observed that the different statistical gene prioritization methods applied to our data prioritized unique and jointly prioritized genes. Therefore, we recommend that investigators incorporate multiple methodologically orthogonal gene prioritization methods to identify a more comprehensive set of causal genes for a given disease. Here, we use two different (orthogonal) expression datasets (BIOS and DEPICT) and three prioritization methods using the same underlying data: MR-IVW, LD-overlap and COLOC. While we believe that the genes prioritized in this study represent robustly prioritized genes for $\mathrm{CeD}$, it is difficult to validate if all the prioritized genes are truly causal based on statistical methodology alone. Functional validation of these genes in disease context is needed to rule out false positives.

The functional validation of TRAFD1 in the siRNA KD experiment in THP-1 cells does establish that this gene regulates the trans-mediated network identified by our eQTL and statistical analysis. Still, the effects of the SCR control and the transfection itself may have obscured some specific TRAFD1-mediated effects. Moreover, the CeD-associated effects of TRAFD1 may not be most pronounced in monocytes or upon LPS-stimulation. Indeed, context- and cell-type-specific effects of CeD-associated genetic variation may hamper the identification of the downstream effects of the prioritized cis- and trans-genes.

In conclusion, this study provides a framework for predicting candidate genes and their function using a systematic in silico approach that could be extended to other complex diseases. Using this approach, we not only confirmed previous association between adaptive cells (gsCD4+ $T$ cells and $B$ cells) and $C e D$, we also unveiled a link between specific genes that may contribute to the disease via innate immune cells, epithelial cells and IE-CTLs. Finally, we found a gene network controlled by TRAFD1 that is part of two major pathways of immune activation, IFN $\gamma$ signalling and $\mathrm{MHC} I$ antigen processing. 
778 Acknowledgements. We are very grateful for the altruistic donation of biological materials 779 by the study participants, without them this study would not be possible. In addition, we 780 thank the UMCG Genomics Coordination center, and the UG Center for Information

781 Technology, and their sponsors BBMRI-NL \& TarGet, for storage and computing 782 infrastructure. We thank BBMRI-NL for providing the transcriptome and genotyped data for 783 the BIOS cohort. We would like to thank Prof. Bana Jabri for providing IE-CTL cell lines, 784 Prof. Morris Swertz for data storage and cluster facilities and Kate Mclntyre for editing the 785 manuscript. This work was supported by an ERC Advanced grant [FP/2007-2013/ERC grant 786 2012-322698] and an NWO Spinoza prize grant [NWO SPI 92-266] to C.W. I.J is supported 787 by a Rosalind Franklin Fellowship from the University of Groningen and an NWO VIDI grant 788 [016.171.047].

790 Data Availability

791 Summary statistics of the CeD GWAS are available from the European Genome-Phenome 792 Archive (https://www.ebi.ac.uk/ega/studies/EGAS00001003805) under accession number 793 EGAS00001003805. The individual-level data of the BIOS cohort is available upon request 794 from https://www.bbmri.nl/acquisition-use-analyze/bios. 
Supplementary Fig. 1 Mediation effect on trans genes for all prioritized genes in the TRAFD1 region on chromosome 12. (A) Three boxes with the eQTL association curves of TRAFD1, SERPING1 and SERPING1 after mediation with TRAFD1. (B) Scatter plot indicating the absolute $Z$ difference between unmediated and mediated trans associations upon mediation ( $\mathrm{y}$ axis) by all mediating cis genes in the TRAFD1 region shown on the $\mathrm{x}$ axis as well as when correcting TRAFD1 expression for the expression of SH2B3 ('TRAFD1 - SH2B3').

Supplementary Fig. 2 TRAFD1 knockdown validation. Cell viability $(\mathbf{A})$ and proliferation $(B)$ of THP-1 cells that were left untransfected (WT) or transfected with non-targeting siRNA (SCR) or siRNA targeting TRAFD1 (KD) for 72 hours. Protein and mRNA levels of TRAFD1 were determined by WB $(\mathbf{C}, \mathbf{D})$ and $\mathrm{QPCR}(\mathbf{E})$. Bars indicate mean \pm SEM. Data are representative of three different experiments. Statistical differences were calculated with a one-sided t-test by using the SCR as $100 \%$ reference. p-value $\left.\leq 0.0001{ }^{* * * *}\right)$. (F) The differential expression analysis approach. Here we compared the gene expression between unstimulated samples and their respective LPS-stimulated samples to identify DEGs that respond to stimulation ( $(|\log 2 \mathrm{FC}|)$ $>1$ and FDR $\leq 0.01$ ). We then identified unique or shared DEGs responding to the stimulation between treatments (WT, SCR or KD), which are shown in two separate Venn diagrams: one for upregulated genes $(\mathbf{G})$ and one for downregulated genes $(\mathbf{H})$.

Supplementary Fig. 3 Expression pattern of TRAFD1-mediated genes upon TRAFD1 knockdown. Heatmap showing the pattern of gene expression of TRAFD1 and of the 41 genes it mediates, scaled by row (see details in Methods and Fig. 5). Expression is shown in different treatments and stimulations as indicated by coloured bars on top of the heatmap.

Supplementary Fig. 4 DEGs upon TRAFD1 knockdown are enriched in TRAFD1-mediated genes. Here we compare the differential expression of 42 genes found in the trans mediation analysis of TRAFD1 (41 trans-mediated genes and TRAFD1) with the differential expression of 42 other randomly chosen genes. The histograms (blue) show the distribution of the median absolute T statistic of DEseq of 42 randomly chosen genes, when 1,000,000 sets of genes are randomly chosen, compared to the observed value for the 42 genes that are from the transmediation analysis (red horizontal line). We compare the results of the control experiment (WTSCR) in panels $\mathbf{A}$ and $\mathbf{C}$ with the results of the knockdown experiment (SCR-KD) in panels $\mathbf{B}$ and D. The fold differences between the control experiments and the knockdown experiments show how much more than expected the 42 genes are differentially expressed in the knockdown compared to the control. 


\section{Supplementary table Legends}

Supplementary Table 1. Prioritization of genes likely causal for celiac disease (CeD). This table contains all the genes in the prioritized CeD regions and their evidence for being causal to CeD. One gene per row is shown. Columns (in order): the human build 37 coordinates of the $\mathrm{CeD}$ region in which the gene is located (region); the gene name according to the ENSEMBL GENES 96 database (human build 37) (gene_name); the ENSEMBL gene identifier (ensembl_id); the most likely effect direction (determined as described in Methods) (most_likely_direction); number of independent eQTL variants found for the gene (n_eqtl_effects); the effect size (MR_ivw_effect) and $p$ value (MR_ivw_p_value) of the MR-IVW test; the summary of LD overlap (Id_overlap_summary), with either the top eQTL variant ('top_snp') or an independent eQTL variant ('cojo_snp') with the r2 linkage disequilibrium between the eQTL SNP and the CeD top variant; the coloc posterior probability of causal variants being shared (coloc_h4); if the gene passes DEPICTs own false discovery thresholds (depict_fdr_pass); and the lines of evidence that are significant compared to the lines of evidence that are available for a gene (lines_of_evidence). Bold fields in any of the columns indicate that the prioritization method is significant according to our thresholds.

Supplementary Table 2. Sensitivity analyses for genes selected by the IVW-MR method. In this table, genes with a significant MR-IVW effect are tested for heterogeneity using the $Q$ test statistic and the MR-weighted median results as sensitivity analysis of all significant MR results. Each row contains the following information: the human build 37 coordinates of the $\mathrm{CeD}$ region in which the gene is located (region); the gene name according to the ENSEMBL GENES 96 database (human build 37) (gene_name); the ENSEMBL gene identifier (ensembl_id); the most likely direction of the effect (determined as described in Methods) (most_likely_direction); the number of independent eQTL variants found (n_eqtl_effects); the effect size (MR_ivw_effect) and $p$ value (MR_ivw_p_value) of the MR-IVW test; the heterogeneity $p$ value of the MR-IVW test using Cochran's Q statistic (MR_heterogeneity_p_value); the weighted median effect estimate (MR_WM_beta) and its associated $p$ value (MR_WM_p); the MR effect estimate after removal of potential outliers (MR_Q_beta); its associated $p$ value $\left(\mathbf{M R} \mathbf{Q} \_\mathbf{p}\right)$; the remaining variants after outlier removal (MR_Q_ivs) and the heterogeneity estimate (MR_Q_heterogeneity).

Supplementary Table 3. Druggability information for prioritized genes. This table contains all the prioritized cis genes in the CeD regions that are existing drug targets according to two different databases (DrugBank v5.1.4, and Finan et al.39). One gene per 
row is shown. Columns indicate (in order): the human build 37 coordinates of the CeD region in which the gene is located (region); the gene name according to the ENSEMBL GENES 96 database (human build 37) (gene_name); the ENSEMBL gene identifier (ensembl_id); the most likely effect direction (determined as described in Methods) (most_likely_direction); the number of independent eQTL variants found for the gene (n_eqtl_effects); the effect size (MR_ivw_effect) and $p$ value (MR_ivw_p_value) of the MR-IVW test; the summary of LD overlap (Id_overlap_summary) with either the top eQTL variant ('top_snp') or an independent eQTL variant ('cojo_snp') with the r2 linkage disequilibrium between the eQTL SNP and the CeD top variant; the coloc posterior probability of causal variants being shared (coloc_h4); if the gene passes DEPICT's own false discovery thresholds (depict_fdr_pass); the lines of evidence that are significant compared to the lines of evidence that are available for a gene (lines_of_evidence); the druggability tier based on Finan et al.39, with lower tiers making it more likely that the gene is druggableзя, (druggable_tier), and if the gene is a drug bank drug target (drug_bank_drug_target). Bold fields in any of the columns indicate that the prioritization method is significant according to our thresholds.

Supplementary Table 4. Cluster assignments for the prioritized genes. The 118 prioritized genes were assigned to a cluster based on a guilt-by-association co-regulation approach to find shared biological mechanisms. For each gene that was prioritized (ensembl_id and gene_name), a cluster membership is given (cluster_membership).

\section{Supplementary Table 5. Significant REACTOME 2016 enrichment of cis prioritized} genes in each co-regulation cluster. Results from the enrichr API using the gene clusters of Supplementary Table 4 as query. Columns indicate: the enrichment background (background); the enrichment term in the background (term_name); the non-corrected $p$ value of enrichment for this term (p_value) and $Z$ score (Z_score); enrichr combined score (combined_score); cis-prioritized genes found in each the term (overlapping_genes) and the multiple testing corrected $p$ value (adjusted_p_value). Each tab of the excel file contain the gene set enrichment for each cluster as defined in Supplementary Table 4.

Supplementary Table 6 . Results of DE analyses from all cell-type- and contextspecific data available for this study (datasets). This table lists all results for the DE analyses (Significant DE genes are defined as padj $<0.05$ and log2 fold change $>|1|$ ) and a summary report of the overlap with TRAFD1 trans-mediated genes (overlap with trans genes+TRAFD1) and relative enrichment. The DE gene lists (padj $<0.05$ and log2 fold change $>|1|)$ for each dataset are given in individual sheets. In the sheet "enrichment", columns upregulated and downregulated indicate if the trans-mediated genes are up- or 
901

902

903

904

905

906

907

908

909

910

911

912

913

914

915

916

917

918

919

920

921

922

923

924

925

926

927

928

929

930

931

932

933

downregulated under stimulated conditions compared to control conditions in each dataset. Enrichment of all the trans mediated genes in the DE genes was determined using a Fisher's exact test and the enrichment $p$ value is shown in the column enrichment $p$-val.

Supplementary Table 7. Functions attributable to the prioritized genes, according to our literature review (see Methods and Fig. 3). Columns describe (in order): gene name (gene_name); ensemble ID (ensembl_id); the change that leads to increased CeD risk, i.e. increased expression (+), decreased expression (-), or undefined (?) (direction); attributable function based in literature (potential_function); and literature or web-based sources (source_1 and source_2). Web-based sources include Gene cards (https://www.genecards.org/) and Genenetwork (https://www.genenetwork.nl/).

\section{Supplementary Table 8. All the significant trans-mediated genes from our cis} prioritization. Each row contains a cis-trans gene pair described with both the ensembl id and hgnc gene name (cis_ensembl_id), (cis_gene_name), (trans_ensembl_id) and (trans_gene_name). Mediation effect and significance are shown using the $Z$ score of the unmediated versus the mediated estimate (using the original unmediated standard error) (z_score_difference) and the mediation $p$ value of the test defined by Friedman and Schatzkin (mediation_p).

Supplementary Table 9. Cell type mediation analysis. We calculated to what extent cell types counted in the BIOS cohort affect the most highly associated TRAFD1 eQTL variant. Columns show (in order): the specific cell type measurements or mediator (mediator); the effect size after mediation by the cell type (mediated_beta); the original effect size (unmediated_beta); difference in effect sizes between mediated and unmediated (beta_difference); the standard error mediation effect size (se); the t-statistic of the beta differences (t_statistic); a $p$ value of the Friedman and Schatzkin test statistic (p_value); the Pearson correlation coefficient between TRAFD1 and the cell type proportion (correlation); and the number of observations in the BIOS cohort (n_observations). If a mediator has a "_Perc" suffix, the cell type counts were converted into ratios. Cell type abbreviations: Baso: Basophil count, EOS: eosinophil count, HCT: haematocrit, HGB: haemoglobin, LUC: large unstained cell count, Lymph: lymphocyte count, $\mathrm{MCH}$ : mean corpuscular haemoglobin, MCHC: mean corpuscular haemoglobin concentration, MCV: mean corpuscular volume, Mono: Monocyte count, MPV: mean platelet volume, Neut: Neutrophil count, PLT: platelets count, RBC: red blood cell count, RDW: red blood cell distribution width, WBC: white blood cell count. 
Supplementary Table 10. Mediation results when correcting TRAFD1 expression for the nearby SH2B3 expression. Columns are: the ENSEMBL id (ensemble_id); the hgnc gene name (gene_name); and the mediation $Z$ score difference ( $Z$ _score_difference), $p$ value (p_value) and Pearson correlation (correlation) between the trans-eQTL top variant and the residual of TRAFD1 expression, after correction for SH2B3 expression.

Supplementary Table 11. Significant REACTOME 2016 enrichment of TRAFD1mediated genes. Results from the enrichr API using the mediated TRAFD1 trans genes as query. Columns indicate: the enrichment background (background); the enrichment term in the background (term_name); the non-corrected $p$ value of enrichment for this term (p_value) and $Z$ score (Z_score); enrichr combined score (combined_score); cisprioritized genes found in each the term (overlapping_genes) and the multiple-testingcorrected $p$ value (adjusted_p_value).

Supplementary Table 12. Differential expression results of the THP-1 experiments. This table shows differential expression analysis of the THP-1 cells with $3 \mathrm{hr}$ LPS treatment (LPS) or without LPS (Unstim) in wild type (WT), scrambled control siRNA (SCR) or TRAFD1 knock down conditions (KD). All conditions and treatments were performed in triplicate. Complete DESEQ2 results are shown for each possible comparison in each tab. For each gene the columns show: the ensembl id per gene (ensembl_id); the mean corrected expression of the gene (baseMean); the log2 fold change of the comparison (log2FoldChange); the standard error of this log2 fold change (IfcSE); a t-statistic of the log2foldchange (stat); the $p$ value (pvalue); and the multiple testing adjusted $p$ value (padj). The direction of the effect is always towards the second term in the tab name: if a log2 fold change is positive and the tab name is, for example, 'WT_LPS_vs_SCR_LPS', then the expression of the gene is increased in the SCR_LPS samples compared to the WT_LPS samples.

Supplementary Table 13. Significant REACTOME 2016 enrichments in genes cells that are significantly upregulated by LPS in all treatments (WT, SCR and KD). Reactome enrichment is shown for genes according in two groups: genes relatively downregulated in the TRAFD1 knockdown experiment (REACTOME_enrichment_cluster1) and genes relatively upregulated in the TRAFD1 knockdown experiment (REACTOME_enrichment_cluster2). Results are shown from the enrichr API analysis using the genes in a cluster as query. Columns indicate: the enrichment background (background); the enrichment term in the background (term_name); the non-corrected $p$ 
968 value of enrichment for this term (p_value) and $Z$ score (Z-score); enrichr combined score 969 (combined_score); cis-prioritized genes found in each the term (overlapping_genes) and 970 the multiple testing corrected $p$ value (adjusted_p_value).

971 


\section{References}

9731 Kuja-Halkola R, Lebwohl B, Halfvarson J, Wijmenga C, Magnusson PKE, Ludvigsson JF. Heritability of non-HLA genetics in coeliac disease: A population-based study in 107000 twins. Gut 2016. doi:10.1136/gutjnl-2016-311713.

9762 Bevan S, Popat S, Braegger CP, Busch A, O'Donoghue D, Falth-Magnusson K et al. 977 Contribution of the MHC region to the familial risk of coeliac disease. J Med Genet $978 \quad 1999 ; 36: 687-690$. genotyping identifies and localizes multiple common and rare variant association signals in celiac disease. Nat Genet 2011; 43: 1193-1201. common variants for celiac disease influencing immune gene expression. Nat Genet 2010; 42: 295-302.

Ricaño-Ponce I, Gutierrez-Achury J, Costa AF, Deelen P, Kurilshikov A, Zorro MM et al. Immunochip meta-analysis in European and Argentinian populations identifies two novel genetic loci associated with celiac disease. Eur J Hum Genet 2019; : 1-11.

Withoff S, Li Y, Jonkers I, Wijmenga C. Understanding Celiac Disease by Genomics. 2016.

Kumar V, Gutierrez-Achury J, Kanduri K, Almeida R, Hrdlickova B, Zhernakova D V. et al. Systematic annotation of celiac disease loci refines pathological pathways and suggests a genetic explanation for increased interferon-gamma levels. Hum $\mathrm{Mol}$ Genet 2015; 24: 397-409.

Ricaño-Ponce I, Zhernakova D V., Deelen P, Luo O, Li X, Isaacs A et al. Refined mapping of autoimmune disease associated genetic variants with gene expression suggests an important role for non-coding RNAs. J Autoimmun 2016; 68: 62-74. analysis of genome-wide association studies in celiac disease and rheumatoid arthritis identifies fourteen non-HLA shared loci. PLoS Genet 2011; 7. doi:10.1371/journal.pgen.1002004. Jonkers $\mathrm{IH}$, Wijmenga $\mathrm{C}$. Context-specific effects of genetic variants associated with autoimmune disease. Hum. Mol. Genet. 2017. doi:10.1093/hmg/ddx254. 
100612 Giambartolomei C, Vukcevic D, Schadt EE, Franke L, Hingorani AD, Wallace C et al.

1007 Bayesian Test for Colocalisation between Pairs of Genetic Association Studies Using Summary Statistics. PLoS Genet 2014; 10: e1004383.

1009

13 Burgess S, Butterworth A, Thompson SG. Mendelian randomization analysis with

1010 multiple genetic variants using summarized data. Genet Epidemiol 2013; 37: 658-

1011 665.

1012

14 Graaf A van der, Claringbould A, Rimbert A, consortium B, Westra H-J, Li Y et al. A novel Mendelian randomization method identifies causal relationships between gene expression and low-density lipoprotein cholesterol levels. bioRxiv 2019; : 671537.

Pers TH, Karjalainen JM, Chan Y, Westra HJ, Wood AR, Yang J et al. Biological interpretation of genome-wide association studies using predicted gene functions. Nat Commun 2015; 6: 5890.

Zhernakova D V., Deelen P, Vermaat M, Van Iterson M, Van Galen M, Arindrarto W et al. Identification of context-dependent expression quantitative trait loci in whole blood. Nat Genet 2017; 49: 139-145. van Greevenbroek MMJ, Jacobs M, van der Kallen CJH, Vermeulen VMMJ, Jansen EHJM, Schalkwijk CG et al. The cross-sectional association between insulin resistance and circulating complement $\mathrm{C} 3$ is partly explained by plasma alanine aminotransferase, independent of central obesity and general inflammation (the CODAM study). Eur J Clin Invest 2011; 41: 372-379.

Deelen J, van den Akker EB, Trompet S, van Heemst D, Mooijaart SP, Slagboom PE et al. Employing biomarkers of healthy ageing for leveraging genetic studies into human longevity. Exp Gerontol 2016; 82: 166-174.

Tigchelaar EF, Zhernakova A, Dekens JAM, Hermes G, Baranska A, Mujagic Z et al. Cohort profile: LifeLines DEEP, a prospective, general population cohort study in the northern Netherlands: Study design and baseline characteristics. BMJ Open 2015; 5: e006772.

Lin BD, Willemsen G, Abdellaoui A, Bartels M, Ehli EA, Davies GE et al. The Genetic Overlap between Hair and Eye Color. Twin Res Hum Genet 2016; 19: 595-599.

1 Huisman MHB, De Jong SW, Van Doormaal PTC, Weinreich SS, Schelhaas HJ, Van Der Kooi AJ et al. Population based epidemiology of amyotrophic lateral sclerosis using capture-recapture methodology. J Neurol Neurosurg Psychiatry 2011; 82: 1165-1170.

2 Hofman A, Brusselle GGO, Murad SD, van Duijn CM, Franco OH, Goedegebure A et 
al. The Rotterdam Study: 2016 objectives and design update. Eur J Epidemiol 2015; 30: 661-708.

23 Võsa U, Claringbould A, Westra H-J, Bonder MJ, Deelen P, Zeng B et al. Unraveling the polygenic architecture of complex traits using blood eQTL metaanalysis. bioRxiv 2018; 18: 447367.

24 Das S, Forer L, Schönherr S, Sidore C, Locke AE, Kwong A et al. Next-generation genotype imputation service and methods. Nat Genet 2016; 48: 1284-1287.

25 Chang CC, Chow CC, Tellier LCAM, Vattikuti S, Purcell SM, Lee JJ. Secondgeneration PLINK: Rising to the challenge of larger and richer datasets. Gigascience 2015; $4: 7$.

26 Dobin A, Davis CA, Schlesinger F, Drenkow J, Zaleski C, Jha S et al. STAR: Ultrafast universal RNA-seq aligner. Bioinformatics 2013; 29: 15-21.

27 Boomsma DI, Wijmenga C, Slagboom EP, Swertz MA, Karssen LC, Abdellaoui A et al. The Genome of the Netherlands: Design, and project goals. Eur J Hum Genet 2014; $22:$ 221-227.

28 Anders S, Pyl PT, Huber W. HTSeq-A Python framework to work with high-throughput sequencing data. Bioinformatics 2015; 31: 166-169.

29 Yang J, Lee SH, Goddard ME, Visscher PM. GCTA: A tool for genome-wide complex trait analysis. Am J Hum Genet 2011; 88: 76-82.

30 Auton A, Abecasis GR, Altshuler DM, Durbin RM, Bentley DR, Chakravarti A et al. A global reference for human genetic variation. Nature 2015; 526: 68-74.

31 Burgess S, Thompson SG. Interpreting findings from Mendelian randomization using the MR-Egger method. Eur J Epidemiol 2017; 32: 377-389.

32 Yang J, Ferreira T, Morris AP, Medland SE, Madden PAF, Heath AC et al. Conditional and joint multiple-SNP analysis of GWAS summary statistics identifies additional variants influencing complex traits. Nat Genet 2012; 44: 369-375.

33 Bowden J, Hemani G, Davey Smith G. Invited Commentary: Detecting Individual and Global Horizontal Pleiotropy in Mendelian Randomization-A Job for the Humble Heterogeneity Statistic? Am J Epidemiol 2018; 187: 2681-2685.

34 Deelen $\mathrm{P}$, van Dam S, Herkert JC, Karjalainen JM, Brugge H, Abbott KM et al. Improving the diagnostic yield of exome- sequencing by predicting gene-phenotype associations using large-scale gene expression analysis. Nat Commun 2019; 10. doi:10.1038/s41467-019-10649-4. 
$1073 \quad 35$ Freedman LS, Schatzkin A. Sample size for studying intermediate endpoints within intervention trials or observational studies. Am J Epidemiol 1992; 136: 1148-1159.

Chen EY, Tan CM, Kou Y, Duan Q, Wang Z, Meirelles GV et al. Enrichr: interactive and collaborative HTML5 gene list enrichment analysis tool. BMC Bioinformatics 2013; $14: 128$.

37 Wishart DS, Feunang YD, Guo AC, Lo EJ, Marcu A, Grant JR et al. DrugBank 5.0: A major update to the DrugBank database for 2018. Nucleic Acids Res 2018; 46: D1074-D1082.

1082

38 Whirl-Carrillo M, McDonagh EM, Hebert JM, Gong L, Sangkuhl K, Thorn CF et al. Pharmacogenomics knowledge for personalized medicine. Clin Pharmacol Ther 2012; 92: 414-417.

39 Finan C, Gaulton A, Kruger FA, Lumbers RT, Shah T, Engmann J et al. The druggable genome and support for target identification and validation in drug development. Sci Transl Med 2017; 9: eaag1166.

Kim D, Langmead B, Salzberg SL. HISAT: a fast spliced aligner with low memory requirements. Nat Methods 2015; 12: 357. Alignment/Map format and SAMtools. Bioinformatics 2009; 25: 2078-2079. Liao Y, Smyth GK, Shi W. The R package Rsubread is easier, faster, cheaper and better for alignment and quantification of RNA sequencing reads. Nucleic Acids Res 2019. doi:10.1093/nar/gkz114.

Anders S, Huber W. Differential expression analysis for sequence count data. Genome Biol 2010; 11: R106.

Zorro MM, Aguirre-Gamboa R, Mayassi T, Ciszewski C, Barisani D, Hu S et al. Tissue alarmins and adaptive cytokine induce dynamic and distinct transcriptional responses in tissue-resident intraepithelial cytotoxic T lymphocytes. J Autoimmun 2020; 108: 102422.

Jabri B, De Serre NPM, Cellier C, Evans K, Gache C, Carvalho C et al. Selective expansion of intraepithelial lymphocytes expressing the HLA-E- specific natural killer receptor CD94 in celiac disease. Gastroenterology 2000; 118: 867-879.

6 Petersen J, Montserrat V, Mujico JR, Loh KL, Beringer DX, Van Lummel M et al. Tcell receptor recognition of HLA-DQ2-gliadin complexes associated with celiac disease. Nat Struct Mol Biol 2014; 21 : 480-488. 
47 Chiu YG, Ritchlin CT. Denosumab: targeting the RANKL pathway to treat rheumatoid arthritis. Expert Opin Biol Ther 2017; 17: 119-128.

48 Lynch JP, Metz DC, Rutgeerts P, Vermeire S, Assche G Van. Biological Therapies for Inflammatory Bowel Diseases. Gastroenterology 2009; 136: 1182-1197.

49 Baldassari LE, Rose JW. Daclizumab: Development, Clinical Trials, and Practical Aspects of Use in Multiple Sclerosis. Neurotherapeutics 2017; 14: 842-858.

50 Goruppi S, Procopio MG, Jo S, Clocchiatti A, Neel V, Dotto GP. The ULK3 Kinase Is Critical for Convergent Control of Cancer-Associated Fibroblast Activation by CSL and GLI. Cell Rep 2017; 20: 2468-2479.

51 Vang T, Abrahamsen H, Myklebust S, Enserink J, Prydz H, Mustelin T et al. Knockdown of $\mathrm{C}$-terminal Src kinase by siRNA-mediated RNA interference augments T cell receptor signaling in mature T cells. Eur J Immunol 2004; 34: 2191-2199.

Abadie V, Discepolo V, Jabri B. Intraepithelial lymphocytes in celiac disease immunopathology. Semin Immunopathol 2012. doi:10.1007/s00281-012-0316-x.

53 Nilsen EM, Lundin KEA, Krajči P, Scott H, Sollid LM, Brandtzaeg P. Gluten specific, HLA-DQ restricted T cells from coeliac mucosa produce cytokines with Th1 or Th0 profile dominated by interferon y. Gut 1995; 37: 766-776.

54 Wapenaar MC, Van Belzen MJ, Fransen JH, Fariña Sarasqueta A, Houwen RHJ, Meijer JWR et al. The interferon gamma gene in celiac disease: Augmented expression correlates with tissue damage but no evidence for genetic susceptibility. $J$ Autoimmun 2004; 23: 183-190.

55 Westra H-J, Martínez-Bonet M, Onengut-Gumuscu S, Lee A, Luo Y, Teslovich N et al. Fine-mapping and functional studies highlight potential causal variants for rheumatoid arthritis and type 1 diabetes. Nat Genet 2018; 50: 1366-1374.

56 Astle WJ, Elding H, Jiang T, Allen D, Ruklisa D, Mann AL et al. The Allelic Landscape of Human Blood Cell Trait Variation and Links to Common Complex Disease. Cell 2016; 167: 1415-1429.e19.

57 Sanada T, Takaesu G, Mashima R, Yoshida R, Kobayashi T, Yoshimura A. FLN29 deficiency reveals its negative regulatory role in the toll-like receptor (TLR) and retinoic acid-inducible gene I (RIG-I)-like helicase signaling pathway. J Biol Chem 2008; 283: 33858-33864.

58 Kim HS, Kim DC, Kim HM, Kwon HJ, Kwon SJ, Kang SJ et al. STAT1 deficiency redirects IFN signalling toward suppression of TLR response through a feedback activation of STAT3. Sci Rep 2015; 5. doi:10.1038/srep13414. 
114059 Majumder S, Bhattacharjee S, Paul Chowdhury B, Majumdar S. CXCL10 Is Critical for the Generation of Protective CD8 T Cell Response Induced by Antigen Pulsed CpGODN Activated Dendritic Cells. PLoS One 2012; 7. doi:10.1371/journal.pone.0048727.

60 Seyffer F, Tampé R. ABC transporters in adaptive immunity. Biochim. Biophys. Acta -

61 Dorrington MG, Fraser IDC. NF-kB signaling in macrophages: Dynamics, crosstalk, and signal integration. Front Immunol 2019; 10: 705.

63 Tye-Din JA, Galipeau HJ, Agardh D. Celiac disease: A review of current concepts in pathogenesis, prevention, and novel therapies. Front Pediatr 2018. doi:10.3389/fped.2018.00350.

65 Linterman MA, Beaton L, Yu D, Ramiscal RR, Srivastava M, Hogan JJ et al. IL-21 acts directly on $\mathrm{B}$ cells to regulate $\mathrm{Bcl}-6$ expression and germinal center responses. $J$ Exp Med 2010. doi:10.1084/jem.20091738.

Høydahl LS, Richter L, Frick R, Snir O, Gunnarsen KS, Landsverk OJB et al. Plasma Cells Are the Most Abundant Gluten Peptide MHC-expressing Cells in Inflamed Intestinal Tissues From Patients With Celiac Disease. Gastroenterology 2019. doi:10.1053/j.gastro.2018.12.013. Jarret A, Jackson R, Duizer C, Healy ME, Zhao J, Rone JM et al. Enteric Nervous System-Derived IL-18 Orchestrates Mucosal Barrier Immunity. Cell 2020. doi:10.1016/j.cell.2019.12.016.

Nowarski R, Jackson R, Gagliani N, De Zoete MR, Palm NW, Bailis W et al. Epithelial IL-18 Equilibrium Controls Barrier Function in Colitis. Cell 2015. doi:10.1016/j.cell.2015.10.072.

9 Mahnke J, Schumacher V, Ahrens S, Käding N, Feldhoff LM, Huber M et al. Interferon Regulatory Factor 4 controls T H1 cell effector function and metabolism. Sci Rep 2016; 6: 35521.

Grenningloh R, Bok YK, Ho IC. Ets-1, a functional cofactor of T-bet, is essential for Th1 inflammatory responses. J Exp Med 2005; 201: 615-626. 
117371 Liu T, Zhang L, Joo D, Sun SC. NF-kB signaling in inflammation. Signal Transduct. $1174 \quad$ Target. Ther. 2017. doi:10.1038/sigtrans.2017.23.

117572 Maiuri MC, De Stefano D, Mele G, Fecarotta S, Greco L, Troncone R et al. Nuclear 1176 factor $\mathrm{KB}$ is activated in small intestinal mucosa of celiac patients. J Mol Med 2003; 81: 373-379.

117873 Fernandez-jimenez N, Castellanos-rubio A, Plaza-izurieta L, Irastorza I, 1179 Elcoroaristizabal X, Jauregi-miguel A et al. Coregulation and modulation of NFkB1180 related genes in celiac disease: Uncovered aspects of gut mucosal inflammation. Hum Mol Genet 2014; 23: 1298-1310.

74 Brynedal B, Choi JM, Raj T, Bjornson R, Stranger BE, Neale BM et al. Large-Scale trans-eQTLs Affect Hundreds of Transcripts and Mediate Patterns of Transcriptional Co-regulation. Am J Hum Genet 2017; 100: 581-591.

Beitnes ACR, Ráki M, Brottveit M, Lundin KEA, Jahnsen FL, Sollid LM. Rapid accumulation of CD14 +CD11C + dendritic cells in gut mucosa of celiac disease after in vivo gluten challenge. PLoS One 2012. doi:10.1371/journal.pone.0033556. Protein Kinase PKR Is Required for Activation of NF-kB by IFN- $\gamma$ in a STAT1Independent Pathway. J Immunol 2001. doi:10.4049/jimmunol.166.10.6170. 
bioRxiv preprint doi: https://doi.org/10.1101/2020.03.04.973487; this version posted March 5, 2020. The copyright holder for this preprint (which was not certified by peer review) is the author/funder. All rights reserved. No reuse allowed without permission. 